DOI 10.4171/JEMS/326

Christian Ausoni · John Rognes

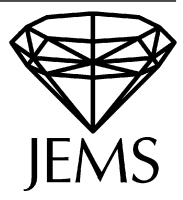

\title{
Algebraic $K$-theory of the first Morava $K$-theory
}

Received November 25, 2009, and in revised form June 17, 2010 and January 27, 2011

Abstract. For a prime $p \geq 5$, we compute the algebraic $K$-theory modulo $p$ and $v_{1}$ of the mod $p$ Adams summand, using topological cyclic homology. On the way, we evaluate its modulo $p$ and $v_{1}$ topological Hochschild homology. Using a localization sequence, we also compute the $K$-theory modulo $p$ and $v_{1}$ of the first Morava $K$-theory.

Keywords. Algebraic $K$-theory, Morava $K$-theory, topological cyclic homology, topological Hochschild homology

\section{Introduction}

In this paper we continue the investigation from [AR02] and [Aus10] of the algebraic $K$ theory of topological $K$-theory and related $S$-algebras. Let $\ell_{p}$ be the $p$-complete Adams summand of connective complex $K$-theory, and let $\ell / p=k(1)$ be the first connective Morava $K$-theory. It has a unique $S$-algebra structure [Ang11, Th. A], and we show in Section 2 that $\ell / p$ is an $\ell_{p}$-algebra (in uncountably many ways), so that $K(\ell / p)$ is a $K\left(\ell_{p}\right)$-module spectrum.

Let $V(1)=S /\left(p, v_{1}\right)$ be the type 2 Smith-Toda complex (see Section 4 below for a definition). It is a homotopy commutative ring spectrum for $p \geq 5$, with a preferred periodic class $v_{2} \in V(1)_{*}$ of degree $2 p^{2}-2$. We write $V(1)_{*}(X)=\pi_{*}(V(1) \wedge X)$ for the $V(1)$-homotopy of a spectrum $X$. Multiplication by $v_{2}$ makes $V(1)_{*}(X)$ a $P\left(v_{2}\right)$-module, where $P\left(v_{2}\right)$ denotes the polynomial algebra over $\mathbb{F}_{p}$ generated by $v_{2}$. We denote by $\mathbb{F}_{p}\left\{x_{1}, \ldots, x_{n}\right\}$ the $\mathbb{F}_{p}$-vector space generated by $x_{1}, \ldots, x_{n}$, and by $E\left(x_{1}, \ldots, x_{n}\right)$ the exterior algebra over $\mathbb{F}_{p}$ generated by $x_{1}, \ldots, x_{n}$.

We computed the $V(1)$-homotopy of $K\left(\ell_{p}\right)$ in [AR02, Th. 9.1], showing that it is essentially a free $P\left(v_{2}\right)$-module on $(4 p+4)$ generators. The following is our main result, corresponding to Theorem 7.7 in the body of the paper.

Ch. Ausoni: Mathematical Institute, University of Münster, DE-48149 Münster, Germany; e-mail: ausoni@uni-muenster.de

J. Rognes: Department of Mathematics, University of Oslo, NO-0316 Oslo, Norway; e-mail: rognes@math.uio.no

Mathematics Subject Classification (2010): 19D55, 55N15 
Theorem 1.1. Let $p \geq 5$ be a prime and let $\ell / p=k(1)$ be the first connective Morava $K$-theory spectrum. There is an isomorphism of $P\left(v_{2}\right)$-modules

$$
\begin{aligned}
V(1)_{*} K(\ell / p) \cong & P\left(v_{2}\right) \otimes E\left(\bar{\epsilon}_{1}\right) \otimes \mathbb{F}_{p}\left\{1, \partial \lambda_{2}, \lambda_{2}, \partial v_{2}\right\} \\
& \oplus P\left(v_{2}\right) \otimes E\left(\operatorname{dlog} v_{1}\right) \otimes \mathbb{F}_{p}\left\{t^{d} v_{2} \mid 0<d<p^{2}-p, p \nmid d\right\} \\
& \oplus P\left(v_{2}\right) \otimes E\left(\bar{\epsilon}_{1}\right) \otimes \mathbb{F}_{p}\left\{t^{d p} \lambda_{2} \mid 0<d<p\right\} .
\end{aligned}
$$

Here $\left|\lambda_{1}\right|=\left|\bar{\epsilon}_{1}\right|=2 p-1,\left|\lambda_{2}\right|=2 p^{2}-1,\left|v_{2}\right|=2 p^{2}-2,\left|\operatorname{dog} v_{1}\right|=1,|\partial|=-1$ and $|t|=-2$. This is a free $P\left(v_{2}\right)$-module of rank $2 p^{2}-2 p+8$ and of zero Euler characteristic.

We prove this theorem by means of the cyclotomic trace map [BHM93] to topological cyclic homology $T C(\ell / p ; p)$. Along the way we evaluate $V(1)_{*} T H H(\ell / p)$, where $T H H$ denotes topological Hochschild homology, as well as $V(1)_{*} T C(\ell / p ; p)$ (see Proposition 4.2 and Theorem 7.6).

Let $L_{p}$ be the $p$-complete Adams summand of periodic complex $K$-theory, and let $L / p=K(1)$ be the first periodic Morava $K$-theory. The localization cofibre sequence $K\left(\mathbb{Z}_{p}\right) \rightarrow K\left(\ell_{p}\right) \rightarrow K\left(L_{p}\right) \rightarrow \Sigma K\left(\mathbb{Z}_{p}\right)$ of Blumberg and Mandell [BM08, p. 157] has the $\bmod p$ Adams analogue

$$
K(\mathbb{Z} / p) \rightarrow K(\ell / p) \rightarrow K(L / p) \rightarrow \Sigma K(\mathbb{Z} / p)
$$

(see Proposition 2.2 below). Using Quillen's computation [Qui72, Th. 7] of $K(\mathbb{Z} / p)$, we obtain the following consequence:

Corollary 1.2. Let $p \geq 5$ be a prime and let $L / p=K(1)$ be the first Morava $K$-theory spectrum. There is an isomorphism of $P\left(v_{2}^{ \pm 1}\right)$-modules

$$
V(1)_{*} K(L / p)\left[v_{2}^{-1}\right] \cong V(1)_{*} K(\ell / p)\left[v_{2}^{-1}\right] .
$$

If there is a class $\operatorname{dlog} v_{1} \in V(1)_{1} K(L / p)$ with $\lambda_{2}=v_{2} \cdot \operatorname{dlog} v_{1}$, then there is an isomorphism of $P\left(v_{2}\right)$-modules

$$
\begin{aligned}
V(1)_{*} K(L / p) \cong & P\left(v_{2}\right) \otimes E\left(\bar{\epsilon}_{1}\right) \otimes \mathbb{F}_{p}\left\{1, \partial \lambda_{2}, \operatorname{dlog} v_{1}, \partial v_{2}\right\} \\
& \oplus P\left(v_{2}\right) \otimes E\left(\operatorname{dlog} v_{1}\right) \otimes \mathbb{F}_{p}\left\{t^{d} v_{2} \mid 0<d<p^{2}-p, p \nmid d\right\} \\
& \oplus P\left(v_{2}\right) \otimes E\left(\bar{\epsilon}_{1}\right) \otimes \mathbb{F}_{p}\left\{t^{d p} v_{2} \operatorname{dlog} v_{1} \mid 0<d<p\right\},
\end{aligned}
$$

where the degrees of the generators are as in Theorem 1.1. This is a free P( $\left.v_{2}\right)$-module of rank $2 p^{2}-2 p+8$ and of zero Euler characteristic.

Our far-reaching aim, which partially motivated the computations presented here, is to conceptually understand the algebraic $K$-theory of $\ell_{p}$ and other commutative $S$-algebras in terms of localization and Galois descent, in the same way as we understand the algebraic $K$-theory of rings of integers in (local) number fields or more general regular rings. The first task is to relate $K\left(\ell_{p}\right)$ to the algebraic $K$-theory of its "residue fields" and "fraction field", for which we expect a description in terms of Galois cohomology to 
exist, starting with the Galois theory for commutative $S$-algebras developed by the second author [Rog08]. The residue rings of $\ell_{p}$ appear to be $\ell / p, H \mathbb{Z}_{p}$ and $H \mathbb{Z} / p$, while the fraction field $f f\left(\ell_{p}\right)$ is more mysterious. For our purposes, its algebraic $K$-theory $K\left(f f\left(\ell_{p}\right)\right)$ should fit in a natural localization cofibre sequence of spectra

$$
K(L / p) \rightarrow K\left(L_{p}\right) \rightarrow K\left(f f\left(\ell_{p}\right)\right) \rightarrow \Sigma K(L / p)
$$

An obvious candidate for $f f\left(\ell_{p}\right)$ is provided by the algebraic localization $L_{p}\left[p^{-1}\right]=$ $L \mathbb{Q}_{p}$, having as coefficients the graded field $\mathbb{Q}_{p}\left[v_{1}^{ \pm 1}\right]$. However, by the following corollary, this is too naive.

Corollary 1.3. The spectra $K(L / p), K\left(L_{p}\right)$ and $K\left(L \mathbb{Q}_{p}\right)$ cannot possibly fit in a cofibre sequence

$$
K(L / p) \rightarrow K\left(L_{p}\right) \rightarrow K\left(L \mathbb{Q}_{p}\right) \rightarrow \Sigma K(L / p)
$$

Indeed, the above computation implies that $V(1)_{*} K(L / p)\left[v_{2}^{-1}\right]$ and $V(1)_{*} K\left(L_{p}\right)\left[v_{2}^{-1}\right]$ are not abstractly isomorphic, while $V(1)_{*} K\left(L \mathbb{Q}_{p}\right)\left[v_{2}^{-1}\right]$ is zero since it is an algebra over $V(1)_{*} K\left(\mathbb{Q}_{p}\right)\left[v_{2}^{-1}\right]=0$. The last equality follows from the computation of the $p$-primary homotopy type of $K\left(\mathbb{Q}_{p}\right)$ [HM03, Th. D], which shows that $V(1)_{*} K\left(\mathbb{Q}_{p}\right)$ is $v_{2}$-torsion.

In conclusion, the conjectural fraction field $f f\left(\ell_{p}\right)$ appears to be a localization of $L_{p}$ away from $L / p$ less drastic than the algebraic localization $L_{p}\left[p^{-1}\right]=L \mathbb{Q}_{p}$. We elaborate more on this issue in [AR].

The paper is organized as follows. In Section 2 we fix our notation, show that $\ell / p$ admits the structure of an associative $\ell_{p}$-algebra, and give a similar discussion for $k u / p$ and the periodic versions $L / p$ and $K U / p$. Section 3 contains the computation of the mod $p$ homology of $T H H(\ell / p)$, and in Section 4 we evaluate its $V(1)$-homotopy. In Section 5 we show that the $C_{p^{n}}$-fixed points and $C_{p^{n}}$-homotopy fixed points of $T H H(\ell / p)$ are closely related, and use this to inductively determine their $V(1)$-homotopy in Section 6. Finally, in Section 7 we achieve the computation of $T C(\ell / p ; p)$ and $K(\ell / p)$ in $V(1)$-homotopy.

Notation and conventions. Let $p$ be a fixed prime. We write $E(x)=\mathbb{F}_{p}[x] /\left(x^{2}\right)$ for the exterior algebra, $P(x)=\mathbb{F}_{p}[x]$ for the polynomial algebra and $P\left(x^{ \pm 1}\right)=\mathbb{F}_{p}\left[x, x^{-1}\right]$ for the Laurent polynomial algebra on one generator $x$, and similarly for a list of generators. We will also write $\Gamma(x)=\mathbb{F}_{p}\left\{\gamma_{i}(x) \mid i \geq 0\right\}$ for the divided power algebra, with $\gamma_{i}(x)$. $\gamma_{j}(x)=(i, j) \gamma_{i+j}(x)$, where $(i, j)=(i+j) ! / i ! j !$ is the binomial coefficient. We use the obvious abbreviations $\gamma_{0}(x)=1$ and $\gamma_{1}(x)=x$. Finally, we write $P_{h}(x)=\mathbb{F}_{p}[x] /\left(x^{h}\right)$ for the truncated polynomial algebra of height $h$, and recall the isomorphism $\Gamma(x) \cong$ $P_{p}\left(\gamma_{p^{e}}(x) \mid e \geq 0\right)$ in characteristic $p$. We write $X_{(p)}$ and $X_{p}$ for the $p$-localization and the $p$-completion, respectively, of any spectrum or abelian group $X$. In the spectral sequences (of $\mathbb{F}_{p}$-modules) discussed below, we often determine differentials only up to multiplication by a unit. We use the notation $d(x) \doteq y$ to indicate that the equation $d(x)=\alpha y$ holds for some unit $\alpha \in \mathbb{F}_{p}$. 


\section{Base change squares of $S$-algebras}

Let $p$ be a prime, even or odd for now. Let $k u$ and $K U$ be the connective and the periodic complex $K$-theory spectra, with homotopy rings $k u_{*}=\mathbb{Z}[u]$ and $K U_{*}=\mathbb{Z}\left[u^{ \pm 1}\right]$, where $|u|=2$. Let $\ell=B P\langle 1\rangle$ and $L=E(1)$ be the $p$-local Adams summands, with $\ell_{*}=$ $\mathbb{Z}_{(p)}\left[v_{1}\right]$ and $L_{*}=\mathbb{Z}_{(p)}\left[v_{1}^{ \pm 1}\right]$, where $\left|v_{1}\right|=2 p-2$. The inclusion $\ell \rightarrow k u_{(p)}$ maps $v_{1}$ to $u^{p-1}$. Alternate notations in the $p$-complete cases are $K U_{p}=E_{1}$ and $L_{p}=\widehat{E(1)}$. These ring spectra are all commutative $S$-algebras, in the sense that each admits a unique $E_{\infty}$ ring spectrum structure. See [BR05, p. 692] for proofs of uniqueness in the periodic cases.

Let $k u / p$ and $K U / p$ be the connective and periodic mod $p$ complex $K$-theory spectra, with coefficients $(k u / p)_{*}=\mathbb{Z} / p[u]$ and $(K U / p)_{*}=\mathbb{Z} / p\left[u^{ \pm 1}\right]$. These are 2-periodic versions of the first Morava $K$-theory spectra $\ell / p=k(1)$ and $L / p=K(1)$, with $(\ell / p)_{*}=\mathbb{Z} / p\left[v_{1}\right]$ and $(L / p)_{*}=\mathbb{Z} / p\left[v_{1}^{ \pm 1}\right]$. Each of these can be constructed as the cofibre of the multiplication by $p$ map, as a module over the corresponding commutative $S$-algebra. For example, there is a cofibre sequence of $k u$-modules $k u \stackrel{p}{\rightarrow} k u \stackrel{i}{\rightarrow} k u / p \rightarrow$ $\Sigma k u$.

Let $H R$ be the Eilenberg-Mac Lane spectrum of a ring $R$. When $R$ is associative, $H R$ admits a unique associative $S$-algebra structure, and when $R$ is commutative, $H R$ admits a unique commutative $S$-algebra structure. The zeroth Postnikov section defines unique maps of commutative $S$-algebras $\pi: k u \rightarrow H \mathbb{Z}$ and $\pi: \ell \rightarrow H \mathbb{Z}_{(p)}$, which can be followed by unique commutative $S$-algebra maps to $H \mathbb{Z} / p$.

The $k u$-module spectrum $k u / p$ does not admit the structure of a commutative $k u$ algebra. It cannot even be an $E_{2}$ or $H_{2}$ ring spectrum, since the homomorphism induced in mod $p$ homology by the resulting map $\pi: k u / p \rightarrow H \mathbb{Z} / p$ of $H_{2}$ ring spectra would not commute with the homology operation $Q^{1}\left(\bar{\tau}_{0}\right)=\bar{\tau}_{1}$ in the target $H_{*}\left(H \mathbb{Z} / p ; \mathbb{F}_{p}\right)$ [BMMS86, III.2.3]. Similar remarks apply for $K U / p, \ell / p$ and $L / p$. Associative algebra structures, or $A_{\infty}$ ring spectrum structures, are easier to come by. The following result is a direct application of the methods of [Laz01, §§9-11]. We adapt the notation of [BJ02, $\S 3]$ to provide some details in our case.

Proposition 2.1. The ku-module spectrum $k u / p$ admits the structure of an associative $k u$-algebra, but the structure is not unique. Similar statements hold for $K U / p$ as a KUalgebra, $\ell / p$ as an $\ell$-algebra and $L / p$ as an L-algebra.

Proof. We construct $k u / p$ as the (homotopy) limit of its Postnikov tower of associative $k u$-algebras $P^{2 m-2}=k u /\left(p, u^{m}\right)$, with coefficient rings $k u /\left(p, u^{m}\right)_{*}=k u_{*} /\left(p, u^{m}\right)$ for $m \geq 1$. To start the induction, $P^{0}=H \mathbb{Z} / p$ is a $k u$-algebra via $i \circ \pi: k u \rightarrow H \mathbb{Z} \rightarrow$ $H \mathbb{Z} / p$. Assume inductively for $m \geq 1$ that $P=P^{2 m-2}$ has been constructed. We will define $P^{2 m}$ by a (homotopy) pullback diagram

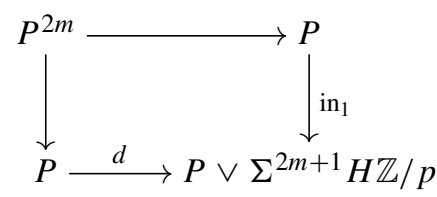


in the category of associative $k u$-algebras. Here

$$
d \in \operatorname{ADer}_{k u}^{2 m+1}(P, H \mathbb{Z} / p) \cong T H H_{k u}^{2 m+2}(P, H \mathbb{Z} / p)
$$

is an associative $k u$-algebra derivation of $P$ with values in $\Sigma^{2 m+1} H \mathbb{Z} / p$, and the group of such can be identified with the indicated topological Hochschild cohomology group of $P$ over $k u$. We recall that these are the homotopy groups (cohomologically graded) of the function spectrum $F_{P \wedge_{k u} P \text { op }}(P, H \mathbb{Z} / p)$. The composite map $\mathrm{pr}_{2} \circ d: P \rightarrow \Sigma^{2 m+1} H \mathbb{Z} / p$ of $k u$-modules, where $\mathrm{pr}_{2}$ projects onto the second wedge summand, is restricted to equal the $k u$-module Postnikov $k$-invariant of $k u / p$ in

$$
H_{k u}^{2 m+1}(P ; \mathbb{Z} / p)=\pi_{0} F_{k u}\left(P, \Sigma^{2 m+1} H \mathbb{Z} / p\right) .
$$

We compute that $\pi_{*}\left(P \wedge_{k u} P^{\text {op }}\right)=k u_{*} /\left(p, u^{m}\right) \otimes E\left(\tau_{0}, \tau_{1, m}\right)$, where $\left|\tau_{0}\right|=1,\left|\tau_{1, m}\right|=$ $2 m+1$ and $E(-)$ denotes the exterior algebra on the given generators. (For $p=2$, the use of the opposite product is essential here [Ang08, §3].) The function spectrum description of topological Hochschild cohomology leads to the spectral sequence

$$
E_{2}^{*, *}=\operatorname{Ext}_{\pi_{*}\left(P \wedge_{k u} P^{\mathrm{op})}\right)}^{*, *}\left(\pi_{*}(P), \mathbb{Z} / p\right) \cong \mathbb{Z} / p\left[y_{0}, y_{1, m}\right] \Rightarrow T H H_{k u}^{*}(P, H \mathbb{Z} / p),
$$

where $y_{0}$ and $y_{1, m}$ have cohomological bidegrees $(1,1)$ and $(1,2 m+1)$, respectively. The spectral sequence collapses at $E_{2}=E_{\infty}$, since it is concentrated in even total degrees. In particular,

$$
\operatorname{ADer}_{k u}^{2 m+1}(P, H \mathbb{Z} / p) \cong \mathbb{F}_{p}\left\{y_{1, m}, y_{0}^{m+1}\right\}
$$

Additively, $H_{k u}^{2 m+1}(P ; \mathbb{Z} / p) \cong \mathbb{F}_{p}\left\{Q_{1, m}\right\}$ is generated by a class dual to $\tau_{1, m}$, which is the image of $y_{1, m}$ under left composition with $\mathrm{pr}_{2}$. It equals the $k u$-module $k$-invariant of $k u / p$. Thus there are precisely $p$ choices $d=y_{1, m}+\alpha y_{0}^{m+1}$, with $\alpha \in \mathbb{F}_{p}$, for how to extend any given associative $k u$-algebra structure on $P=P^{2 m-2}$ to one on $P^{2 m}=$ $k u /\left(p, u^{m+1}\right)$. In the limit, we find that there are an uncountable number of associative $k u$-algebra structures on $k u / p=\operatorname{holim}_{m} P^{2 m}$, each indexed by a sequence of choices $\alpha \in \mathbb{F}_{p}$ for all $m \geq 1$.

The periodic spectrum $K U / p$ can be obtained from $k u / p$ by Bousfield $K U$-localization in the category of $k u$-modules [EKMM97, VIII.4], which makes it an associative $K U$ algebra. The classification of periodic $S$-algebra structures is the same as in the connective case, since the original $k u$-algebra structure on $k u / p$ can be recovered from that on $K U / p$ by a functorial passage to the connective cover. To construct $\ell / p$ as an associative $\ell$ algebra, or $L / p$ as an associative $L$-algebra, replace $u$ by $v_{1}$ in these arguments.

By varying the ground $S$-algebra, we obtain the same conclusions about $k u / p$ as a $k u_{(p)^{-}}$ algebra or $k u_{p}$-algebra, and about $\ell / p$ as an $\ell_{p}$-algebra.

For each choice of $k u$-algebra structure on $k u / p$, the zeroth Postnikov section

$$
\pi: k u / p \rightarrow H \mathbb{Z} / p
$$


is a $k u$-algebra map, with the unique $k u$-algebra structure on the target. Hence there is a commutative square of associative $k u$-algebras

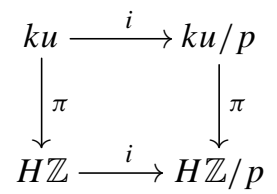

and similarly in the $p$-local and $p$-complete cases. In view of the weak equivalence $H \mathbb{Z} \wedge_{k u} k u / p \simeq H \mathbb{Z} / p$, this square expresses the associative $H \mathbb{Z}$-algebra $H \mathbb{Z} / p$ as the base change of the associative $k u$-algebra $k u / p$ along $\pi: k u \rightarrow H \mathbb{Z}$. Likewise, there is a commutative square of associative $\ell_{p}$-algebras

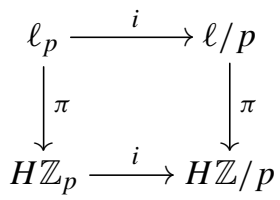

that expresses $H \mathbb{Z} / p$ as the base change of $\ell / p$ along $\ell_{p} \rightarrow H \mathbb{Z}_{p}$, and similarly in the $p$-local case. By omission of structure, these squares are also diagrams of $S$-algebras and $S$-algebra maps.

We end this section by formulating the $\bmod p$ analogue of the localization cofibre sequence in algebraic $K$-theory

$$
K\left(\mathbb{Z}_{p}\right) \rightarrow K\left(\ell_{p}\right) \rightarrow K\left(L_{p}\right) \rightarrow \Sigma K\left(\mathbb{Z}_{p}\right)
$$

conjectured by the second author and established by Blumberg and Mandell [BM08, p. 157].

Proposition 2.2. There is a localization cofibre sequence of spectra

$$
K(\mathbb{Z} / p) \rightarrow K(\ell / p) \rightarrow K(L / p) \rightarrow \Sigma K(\mathbb{Z} / p)
$$

where the first map is the transfer and the second map is induced by the localization $\ell / p \rightarrow \ell / p\left[v_{1}^{-1}\right]=L / p$.

Proof. The proof of the existence of the localization sequence (2.2) given in [BM08, pp. 160-163] and the identification of the transfer map adapt without change to cover the $\bmod p$ analogue stated in this proposition. Here we use that a finite cell $\ell / p$-module that is $v_{1}$-torsion has finite homotopy groups, and the nonzero groups are concentrated in a finite range of degrees.

\section{Topological Hochschild homology}

We shall compute the $V(1)$-homotopy of the topological Hochschild homology $T H H(-)$ and topological cyclic homology $T C(-; p)$ of the $S$-algebras in diagram (2.1), for primes $p \geq 5$. Passing to connective covers, this also computes the $V(1)$-homotopy of the alge- 
braic $K$-theory spectra appearing in that square. With these coefficients, or more generally, after $p$-adic completion, the functors $T H H$ and $T C$ are insensitive to $p$-completion in the argument, so we shall simplify the notation slightly by working with the associative $S$-algebras $\ell$ and $H \mathbb{Z}_{(p)}$ in place of $\ell_{p}$ and $H \mathbb{Z}_{p}$. For ordinary rings $R$ we almost always shorten notations like $\mathrm{THH}(H R)$ to $\mathrm{THH}(R)$.

The computations follow the strategy of [Bök], [BM94], [BM95] and [HM97] for $H \mathbb{Z} / p$ and $H \mathbb{Z}$, and of [MS93] and [AR02] for $\ell$. See also [AR05, §§4-7] for further discussion of the $T H H$-part of such computations. In this section we shall compute the mod $p$ homology of the topological Hochschild homology of $\ell / p$ as a module over the corresponding homology for $\ell$, for any odd prime $p$.

Remark 3.1. Our computations are based on comparisons, using the maps displayed in diagram (2.1) above. We will abuse notation and use the same name for classes in the homology or $V(1)$-homotopy of $T H H\left(\ell_{p}\right), T H H(\ell / p), T H H\left(\mathbb{Z}_{p}\right)$ or $T H H(\mathbb{Z} / p)$, when these classes unambiguously correspond to each other under the homomorphisms induced by the maps $i$ and $\pi$ in (2.1). We also use this abuse of notation in later sections for the $V(1)$-homotopy of $T C$, etc.

We write $H_{*}(-)$ for homology with $\bmod p$ coefficients. It takes values in graded $A_{*}$-comodules, where $A_{*}$ is the dual Steenrod algebra [Mil58, Th 2]. Explicitly (for $p$ odd),

$$
A_{*}=P\left(\bar{\xi}_{k} \mid k \geq 1\right) \otimes E\left(\bar{\tau}_{k} \mid k \geq 0\right)
$$

with coproduct

$$
\psi\left(\bar{\xi}_{k}\right)=\sum_{i+j=k} \bar{\xi}_{i} \otimes \bar{\xi}_{j}^{p^{i}} \quad \text { and } \quad \psi\left(\bar{\tau}_{k}\right)=1 \otimes \bar{\tau}_{k}+\sum_{i+j=k} \bar{\tau}_{i} \otimes \bar{\xi}_{j}^{p^{i}}
$$

Here $\bar{\xi}_{0}=1, \bar{\xi}_{k}=\chi\left(\xi_{k}\right)$ has degree $2\left(p^{k}-1\right)$ and $\bar{\tau}_{k}=\chi\left(\tau_{k}\right)$ has degree $2 p^{k}-1$, where $\chi$ is the canonical conjugation [MM65, 8.4]. Then the maps $i$ and the zeroth Postnikov sections $\pi$ of (2.1) induce identifications

$$
\begin{aligned}
H_{*}\left(H \mathbb{Z}_{(p)}\right) & =P\left(\bar{\xi}_{k} \mid k \geq 1\right) \otimes E\left(\bar{\tau}_{k} \mid k \geq 1\right), \\
H_{*}(\ell) & =P\left(\bar{\xi}_{k} \mid k \geq 1\right) \otimes E\left(\bar{\tau}_{k} \mid k \geq 2\right), \\
H_{*}(\ell / p) & =P\left(\bar{\xi}_{k} \mid k \geq 1\right) \otimes E\left(\bar{\tau}_{0}, \bar{\tau}_{k} \mid k \geq 2\right)
\end{aligned}
$$

as $A_{*}$-comodule subalgebras of $H_{*}(H \mathbb{Z} / p)=A_{*}$. We often make use of the following $A_{*}$-comodule coactions:

$$
\begin{array}{ll}
\nu\left(\bar{\tau}_{0}\right)=1 \otimes \bar{\tau}_{0}+\bar{\tau}_{0} \otimes 1, & v\left(\bar{\xi}_{1}\right)=1 \otimes \bar{\xi}_{1}+\bar{\xi}_{1} \otimes 1, \\
\nu\left(\bar{\tau}_{1}\right)=1 \otimes \bar{\tau}_{1}+\bar{\tau}_{0} \otimes \bar{\xi}_{1}+\bar{\tau}_{1} \otimes 1, & v\left(\bar{\xi}_{2}\right)=1 \otimes \bar{\xi}_{2}+\bar{\xi}_{1} \otimes \bar{\xi}_{1}^{p}+\bar{\xi}_{2} \otimes 1, \\
\nu\left(\bar{\tau}_{2}\right)=1 \otimes \bar{\tau}_{2}+\bar{\tau}_{0} \otimes \bar{\xi}_{2}+\bar{\tau}_{1} \otimes \bar{\xi}_{1}^{p}+\bar{\tau}_{2} \otimes 1 . &
\end{array}
$$

The Bökstedt spectral sequences

$$
E^{2}(B)=H H_{*}\left(H_{*}(B)\right) \Rightarrow H_{*}(T H H(B))
$$


for the commutative $S$-algebras $B=H \mathbb{Z} / p, H \mathbb{Z}_{(p)}$ and $\ell$ begin

$$
\begin{aligned}
E^{2}(\mathbb{Z} / p) & =A_{*} \otimes E\left(\sigma \bar{\xi}_{k} \mid k \geq 1\right) \otimes \Gamma\left(\sigma \bar{\tau}_{k} \mid k \geq 0\right), \\
E^{2}\left(\mathbb{Z}_{(p)}\right) & =H_{*}\left(H \mathbb{Z}_{(p)}\right) \otimes E\left(\sigma \bar{\xi}_{k} \mid k \geq 1\right) \otimes \Gamma\left(\sigma \bar{\tau}_{k} \mid k \geq 1\right), \\
E^{2}(\ell) & =H_{*}(\ell) \otimes E\left(\sigma \bar{\xi}_{k} \mid k \geq 1\right) \otimes \Gamma\left(\sigma \bar{\tau}_{k} \mid k \geq 2\right) .
\end{aligned}
$$

Here $H H_{*}\left(H_{*}(B)\right)$ denotes the Hochschild homology of the graded $\mathbb{F}_{p}$-algebra $H_{*}(B)$. In the above formula we made use of the $\mathbb{F}_{p}$-linear operator $\sigma: H_{*}(B) \rightarrow H H_{1}\left(H_{*}(B)\right)$, $x \mapsto \sigma x$, where $\sigma x$ is the class represented by $1 \otimes x-x \otimes 1$ in the Hochschild complex. Notice that $\sigma$ is the restriction of Connes' operator $d$ to $H H_{0}\left(H_{*}(B)\right)=H_{*}(B)$, and is a derivation in the sense that

$$
\sigma(x y)=x \sigma(y)+(-1)^{|x||y|} y \sigma(x)
$$

for all $x, y \in H_{*}(B)$. These spectral sequences are (graded) commutative $A_{*}$-comodule algebra spectral sequences, and there are differentials

$$
d^{p-1}\left(\gamma_{j} \sigma \bar{\tau}_{k}\right) \doteq \sigma \bar{\xi}_{k+1} \cdot \gamma_{j-p} \sigma \bar{\tau}_{k}
$$

for $j \geq p$ and $k \geq 0$ (see [Bök, Lem. 1.3], [Hun96, Th. 1] or [Aus05, Lem. 5.3]), leaving

$$
\begin{aligned}
E^{\infty}(\mathbb{Z} / p) & =A_{*} \otimes P_{p}\left(\sigma \bar{\tau}_{k} \mid k \geq 0\right), \\
E^{\infty}\left(\mathbb{Z}_{(p)}\right) & =H_{*}\left(H \mathbb{Z}_{(p)}\right) \otimes E\left(\sigma \bar{\xi}_{1}\right) \otimes P_{p}\left(\sigma \bar{\tau}_{k} \mid k \geq 1\right), \\
E^{\infty}(\ell) & =H_{*}(\ell) \otimes E\left(\sigma \bar{\xi}_{1}, \sigma \bar{\xi}_{2}\right) \otimes P_{p}\left(\sigma \bar{\tau}_{k} \mid k \geq 2\right) .
\end{aligned}
$$

The inclusion of 0 -simplices $\eta: B \rightarrow T H H(B)$ is split for commutative $B$ by the augmentation $\epsilon: \operatorname{THH}(B) \rightarrow B$. Thus there are unique representatives in Bökstedt filtration 1, with zero augmentation, for each of the classes $\sigma x$. There are multiplicative extensions $\left(\sigma \bar{\tau}_{k}\right)^{p}=\sigma \bar{\tau}_{k+1}$ for $k \geq 0$ (see [AR05, Prop. 5.9]), so

$$
\begin{aligned}
H_{*}(T H H(\mathbb{Z} / p)) & =A_{*} \otimes P\left(\sigma \bar{\tau}_{0}\right), \\
H_{*}\left(T H H\left(\mathbb{Z}_{(p)}\right)\right) & =H_{*}\left(H \mathbb{Z}_{(p)}\right) \otimes E\left(\sigma \bar{\xi}_{1}\right) \otimes P\left(\sigma \bar{\tau}_{1}\right), \\
H_{*}(T H H(\ell)) & =H_{*}(\ell) \otimes E\left(\sigma \bar{\xi}_{1}, \sigma \bar{\xi}_{2}\right) \otimes P\left(\sigma \bar{\tau}_{2}\right)
\end{aligned}
$$

as $A_{*}$-comodule algebras. The $A_{*}$-comodule coactions are given by

$$
\begin{array}{ll}
\nu\left(\sigma \bar{\tau}_{0}\right)=1 \otimes \sigma \bar{\tau}_{0}, & v\left(\sigma \bar{\xi}_{1}\right)=1 \otimes \sigma \bar{\xi}_{1}, \\
\nu\left(\sigma \bar{\tau}_{1}\right)=1 \otimes \sigma \bar{\tau}_{1}+\bar{\tau}_{0} \otimes \sigma \bar{\xi}_{1}, & v\left(\sigma \bar{\xi}_{2}\right)=1 \otimes \sigma \bar{\xi}_{2}, \\
\nu\left(\sigma \bar{\tau}_{2}\right)=1 \otimes \sigma \bar{\tau}_{2}+\bar{\tau}_{0} \otimes \sigma \bar{\xi}_{2} . &
\end{array}
$$

The natural map $\pi_{*}: T H H(\ell) \rightarrow T H H\left(\mathbb{Z}_{(p)}\right)$ induced by $\pi: \ell \rightarrow \mathbb{Z}_{(p)}$ takes $\sigma \bar{\xi}_{2}$ to 0 and $\sigma \bar{\tau}_{2}$ to $\left(\sigma \bar{\tau}_{1}\right)^{p}$. The natural map $i_{*}: T H H\left(\mathbb{Z}_{(p)}\right) \rightarrow T H H(\mathbb{Z} / p)$ induced by $i: \mathbb{Z}_{(p)} \rightarrow$ $\mathbb{Z} / p$ takes $\sigma \bar{\xi}_{1}$ to 0 and $\sigma \bar{\tau}_{1}$ to $\left(\sigma \bar{\tau}_{0}\right)^{p}$.

The Bökstedt spectral sequence for the associative $S$-algebra $B=\ell / p$ begins

$$
E^{2}(\ell / p)=H_{*}(\ell / p) \otimes E\left(\sigma \bar{\xi}_{k} \mid k \geq 1\right) \otimes \Gamma\left(\sigma \bar{\tau}_{0}, \sigma \bar{\tau}_{k} \mid k \geq 2\right) .
$$


It is an $A_{*}$-comodule module spectral sequence over the Bökstedt spectral sequence for $\ell$, since the $\ell$-algebra multiplication $\ell \wedge \ell / p \rightarrow \ell / p$ is a map of associative $S$-algebras. However, it is not itself an algebra spectral sequence, since the product on $\ell / p$ is not commutative enough to induce a natural product structure on $\operatorname{THH}(\ell / p)$. Nonetheless, we will use the algebra structure present at the $E^{2}$-term to help in naming classes.

The map $\pi: \ell / p \rightarrow H \mathbb{Z} / p$ induces an injection of Bökstedt spectral sequence $E^{2}$ terms, so there are differentials generated algebraically by

$$
d^{p-1}\left(\gamma_{j} \sigma \bar{\tau}_{k}\right) \doteq \sigma \bar{\xi}_{k+1} \cdot \gamma_{j-p} \sigma \bar{\tau}_{k}
$$

for $j \geq p, k=0$ or $k \geq 2$, leaving

$$
E^{\infty}(\ell / p)=H_{*}(\ell / p) \otimes E\left(\sigma \bar{\xi}_{2}\right) \otimes P_{p}\left(\sigma \bar{\tau}_{0}, \sigma \bar{\tau}_{k} \mid k \geq 2\right)
$$

as an $A_{*}$-comodule module over $E^{\infty}(\ell)$. In order to obtain $H_{*}(T H H(\ell / p))$, we need to resolve the $A_{*}$-comodule and $H_{*}(T H H(\ell))$-module extensions. This is achieved in Lemma 3.3 below.

The natural map $\pi_{*}: E^{\infty}(\ell / p) \rightarrow E^{\infty}(\mathbb{Z} / p)$ is an isomorphism in total degrees $\leq 2 p-2$ and injective in total degrees $\leq 2 p^{2}-2$. The first class in the kernel is $\sigma \bar{\xi}_{2}$. Hence there are unique classes

$$
1, \bar{\tau}_{0}, \sigma \bar{\tau}_{0}, \bar{\tau}_{0} \sigma \bar{\tau}_{0}, \ldots,\left(\sigma \bar{\tau}_{0}\right)^{p-1}
$$

in degrees $0 \leq * \leq 2 p-2$ of $H_{*}(T H H(\ell / p))$, mapping to classes with the same names in $H_{*}(T H H(\overline{\mathbb{Z}} / p))$. More concisely, these are the monomials $\bar{\tau}_{0}^{\delta}\left(\sigma \bar{\tau}_{0}\right)^{i}$ for $0 \leq \delta \leq 1$ and $0 \leq i \leq p-1$, except that the degree $2 p-1$ case $(\delta, i)=(1, p-1)$ is omitted. The $A_{*}$-comodule coaction on these classes is given by the same formulas in $H_{*}(T H H(\ell / p))$ as in $H_{*}(T H H(\mathbb{Z} / p))$, cf. (3.1).

There is also a class $\bar{\xi}_{1}$ in degree $2 p-2$ of $H_{*}(T H H(\ell / p))$ mapping to a class with the same name, and same $A_{*}$-coaction, in $H_{*}(T H H(\mathbb{Z} / p))$.

In degree $2 p-1, \pi_{*}$ is a map of extensions from

$$
0 \rightarrow \mathbb{F}_{p}\left\{\bar{\xi}_{1} \bar{\tau}_{0}\right\} \rightarrow H_{2 p-1}(T H H(\ell / p)) \rightarrow \mathbb{F}_{p}\left\{\bar{\tau}_{0}\left(\sigma \bar{\tau}_{0}\right)^{p-1}\right\} \rightarrow 0
$$

to

$$
0 \rightarrow \mathbb{F}_{p}\left\{\bar{\tau}_{1}, \bar{\xi}_{1} \bar{\tau}_{0}\right\} \rightarrow H_{2 p-1}(T H H(\mathbb{Z} / p)) \rightarrow \mathbb{F}_{p}\left\{\bar{\tau}_{0}\left(\sigma \bar{\tau}_{0}\right)^{p-1}\right\} \rightarrow 0
$$

The latter extension is canonically split by the augmentation $\epsilon: T H H(\mathbb{Z} / p) \rightarrow H \mathbb{Z} / p$, which uses the commutativity of the $S$-algebra $H \mathbb{Z} / p$.

In degree $2 p$, the map $\pi_{*}$ goes from

$$
H_{2 p}(T H H(\ell / p))=\mathbb{F}_{p}\left\{\bar{\xi}_{1} \sigma \bar{\tau}_{0}\right\}
$$

to

$$
0 \rightarrow \mathbb{F}_{p}\left\{\bar{\tau}_{0} \bar{\tau}_{1}\right\} \rightarrow H_{2 p}(T H H(\mathbb{Z} / p)) \rightarrow \mathbb{F}_{p}\left\{\sigma \bar{\tau}_{1}, \bar{\xi}_{1} \sigma \bar{\tau}_{0}\right\} \rightarrow 0 .
$$

Again the last extension is canonically split. 
Lemma 3.2. There is a unique class y in $H_{2 p-1}(T H H(\ell / p))$ represented by $\bar{\tau}_{0}\left(\sigma \bar{\tau}_{0}\right)^{p-1}$ in $E_{p-1, p}^{\infty}(\ell / p)$ and mapped by $\pi_{*}$ to $\bar{\tau}_{0}\left(\sigma \bar{\tau}_{0}\right)^{p-1}-\bar{\tau}_{1}$ in $H_{*}(T H H(\mathbb{Z} / p))$.

Proof. This follows from naturality of the suspension operator $\sigma$ and the multiplicative relation $\left(\sigma \bar{\tau}_{0}\right)^{p}=\sigma \bar{\tau}_{1}$ in $H_{*}(T H H(\mathbb{Z} / p))$. A class $y$ in $H_{2 p-1}(T H H(\ell / p))$ represented by $\bar{\tau}_{0}\left(\sigma \bar{\tau}_{0}\right)^{p-1}$ is determined modulo $\bar{\xi}_{1} \bar{\tau}_{0}$. Its image in $H_{2 p-1}(T H H(\mathbb{Z} / p))$ thus has the form $\alpha \bar{\tau}_{1}+\bar{\tau}_{0}\left(\sigma \bar{\tau}_{0}\right)^{p-1}$ modulo $\bar{\xi}_{1} \bar{\tau}_{0}$, for some $\alpha \in \mathbb{F}_{p}$. The suspension $\sigma y$ lies in $H_{2 p}(T H H(\ell / p))=\mathbb{F}_{p}\left\{\bar{\xi}_{1} \sigma \bar{\tau}_{0}\right\}$, so its image in $H_{2 p}(T H H(\mathbb{Z} / p))$ is 0 modulo $\bar{\tau}_{0} \bar{\tau}_{1}$ and $\bar{\xi}_{1} \sigma \bar{\tau}_{0}$. It is also the suspension of $\alpha \bar{\tau}_{1}+\bar{\tau}_{0}\left(\sigma \bar{\tau}_{0}\right)^{p-1}$ modulo $\bar{\xi}_{1} \bar{\tau}_{0}$, which equals $\sigma\left(\alpha \bar{\tau}_{1}\right)+\left(\sigma \bar{\tau}_{0}\right)^{p}=(\alpha+1) \sigma \bar{\tau}_{1}$. In particular, the coefficient $\alpha+1$ of $\sigma \bar{\tau}_{1}$ is 0 , so $\alpha=-1$.

Let

$$
H_{*}(T H H(\ell)) /\left(\sigma \bar{\xi}_{1}\right)=H_{*}(\ell) \otimes E\left(\sigma \bar{\xi}_{2}\right) \otimes P\left(\sigma \bar{\tau}_{2}\right)
$$

denote the quotient algebra of $H_{*}(T H H(\ell))$ by the ideal generated by $\sigma \bar{\xi}_{1}$.

Lemma 3.3. The classes

$$
1, \bar{\tau}_{0}, \sigma \bar{\tau}_{0}, \bar{\tau}_{0} \sigma \bar{\tau}_{0}, \ldots,\left(\sigma \bar{\tau}_{0}\right)^{p-1}, \bar{\tau}_{0}\left(\sigma \bar{\tau}_{0}\right)^{p-1},
$$

in $E^{\infty}(\ell / p)$ represent unique homology classes in $H_{*}(T H H(\ell / p))$, which by abuse of notation will be denoted

$$
1, \bar{\tau}_{0}, \sigma \bar{\tau}_{0}, \bar{\tau}_{0} \sigma \bar{\tau}_{0}, \ldots,\left(\sigma \bar{\tau}_{0}\right)^{p-1}, y,
$$

mapping under $\pi_{*}$ to classes with the same names in $H_{*}(T H H(\mathbb{Z} / p))$, except for $y$, which maps to

$$
\bar{\tau}_{0}\left(\sigma \bar{\tau}_{0}\right)^{p-1}-\bar{\tau}_{1}
$$

The graded $H_{*}(T H H(\ell))$-module $H_{*}(T H H(\ell / p))$ is a free $H_{*}(T H H(\ell)) /\left(\sigma \bar{\xi}_{1}\right)$-module of rank $2 p$ generated by these classes in degrees 0 through $2 p-1$ :

$$
H_{*}(T H H(\ell / p))=H_{*}(T H H(\ell)) /\left(\sigma \bar{\xi}_{1}\right) \otimes \mathbb{F}_{p}\left\{1, \bar{\tau}_{0}, \sigma \bar{\tau}_{0}, \bar{\tau}_{0} \sigma \bar{\tau}_{0}, \ldots,\left(\sigma \bar{\tau}_{0}\right)^{p-1}, y\right\} .
$$

The $A_{*}$-comodule coactions are given by

$$
\begin{aligned}
v\left(\left(\sigma \bar{\tau}_{0}\right)^{i}\right) & =1 \otimes\left(\sigma \bar{\tau}_{0}\right)^{i} \quad \text { for } 0 \leq i \leq p-1, \\
v\left(\bar{\tau}_{0}\left(\sigma \bar{\tau}_{0}\right)^{i}\right) & =1 \otimes \bar{\tau}_{0}\left(\sigma \bar{\tau}_{0}\right)^{i}+\bar{\tau}_{0} \otimes\left(\sigma \bar{\tau}_{0}\right)^{i} \quad \text { for } 0 \leq i \leq p-2, \\
v(y) & =1 \otimes y+\bar{\tau}_{0} \otimes\left(\sigma \bar{\tau}_{0}\right)^{p-1}-\bar{\tau}_{0} \otimes \bar{\xi}_{1}-\bar{\tau}_{1} \otimes 1 .
\end{aligned}
$$

Proof. $H_{*}(\ell / p)$ is freely generated as a module over $H_{*}(\ell)$ by 1 and $\bar{\tau}_{0}$, and the classes $\sigma \bar{\xi}_{2}$ and $\sigma \bar{\tau}_{2}$ in $H_{*}(T H H(\ell))$ induce multiplication by the same symbols in $E^{\infty}(\ell / p)$, as given in (3.2). This generates all of $E^{\infty}(\ell / p)$ from the $2 p$ classes $\bar{\tau}_{0}^{\delta}\left(\sigma \bar{\tau}_{0}\right)^{i}$ for $0 \leq \delta \leq 1$ and $0 \leq i \leq p-1$.

We claim that multiplication by $\sigma \bar{\xi}_{1}$ acts trivially on $H_{*}(T H H(\ell / p))$. It suffices to verify this on the module generators $\bar{\tau}_{0}^{\delta}\left(\sigma \bar{\tau}_{0}\right)^{i}$, for which the product with $\sigma \bar{\xi}_{1}$ remains in the range of degrees where the map to $H_{*}(T H H(\mathbb{Z} / p))$ is injective. The action of $\sigma \bar{\xi}_{1}$ is 
trivial on $H_{*}(T H H(\mathbb{Z} / p))$, since $d^{p-1}\left(\gamma_{p} \sigma \bar{\tau}_{0}\right) \doteq \sigma \bar{\xi}_{1}$ and $\epsilon\left(\sigma \bar{\xi}_{1}\right)=0$, and this implies the claim.

The $A_{*}$-comodule coaction on each module generator, including $y$, is determined by that on its image under $\pi_{*}$. In the latter case, for example, we have

$$
\begin{aligned}
\left(1 \otimes \pi_{*}\right)(v(y)) & =v\left(\pi_{*}(y)\right)=v\left(\bar{\tau}_{0}\left(\sigma \bar{\tau}_{0}\right)^{p-1}-\bar{\tau}_{1}\right) \\
& =1 \otimes \bar{\tau}_{0}\left(\sigma \bar{\tau}_{0}\right)^{p-1}+\bar{\tau}_{0} \otimes\left(\sigma \bar{\tau}_{0}\right)^{p-1}-1 \otimes \bar{\tau}_{1}-\bar{\tau}_{0} \otimes \bar{\xi}_{1}-\bar{\tau}_{1} \otimes 1 \\
& =\left(1 \otimes \pi_{*}\right)\left(1 \otimes y+\bar{\tau}_{0} \otimes\left(\sigma \bar{\tau}_{0}\right)^{p-1}-\bar{\tau}_{0} \otimes \bar{\xi}_{1}-\bar{\tau}_{1} \otimes 1\right),
\end{aligned}
$$

and this proves our formula for $v(y)$ since $1 \otimes \pi_{*}$ is injective in this degree.

Remark 3.4. Notice that Lemma 3.3 implies that for different choices of $\ell$-module structure on $\ell / p$, the resulting homology groups $H_{*}(T H H(\ell / p))$ are (abstractly) isomorphic as graded $H_{*}(T H H(\ell))$-modules and $A_{*}$-comodules.

\section{Passage to $V(1)$-homotopy}

For $p \geq 5$ the Smith-Toda complex $V(1)=S \cup_{p} e^{1} \cup_{\alpha_{1}} e^{2 p-1} \cup_{p} e^{2 p}$ is a homotopy commutative ring spectrum [Smi70, Th. 5.1], [Oka84, Ex. 4.5]. It is defined as the mapping cone of the Adams self-map $v_{1}: \Sigma^{2 p-2} V(0) \rightarrow V(0)$ of the mod $p$ Moore spectrum $V(0)=S \cup_{p} e^{1}$. Hence there is a cofibre sequence

$$
\Sigma^{2 p-2} V(0) \stackrel{v_{1}}{\rightarrow} V(0) \stackrel{i_{1}}{\rightarrow} V(1) \stackrel{j_{1}}{\rightarrow} \Sigma^{2 p-1} V(0) .
$$

There are some choices of orientations involved in fixing such an exact triangle (cf. for instance [HM03, Sect. 2.1]). The composite map $\beta_{1,1}=i_{1} j_{1}: V(1) \rightarrow \Sigma^{2 p-1} V(1)$ defines the primary $v_{1}$-Bockstein homomorphism, acting naturally on $V(1)_{*}(X)$.

In this section we compute $V(1)_{*} T H H(\ell / p)$ as a module over $V(1)_{*} T H H(\ell)$, for any prime $p \geq 5$. The unique ring spectrum map from $V(1)$ to $H \mathbb{Z} / p$ induces the identification

$$
H_{*}(V(1))=E\left(\tau_{0}, \tau_{1}\right)
$$

(no conjugations) as $A_{*}$-comodule subalgebras of $A_{*}$ (see [Tod71, §4]). Here

$$
\nu\left(\tau_{0}\right)=1 \otimes \tau_{0}+\tau_{0} \otimes 1, \quad \nu\left(\tau_{1}\right)=1 \otimes \tau_{1}+\xi_{1} \otimes \tau_{0}+\tau_{1} \otimes 1 .
$$

A form of the following lemma goes back to [Whi62, p. 271].

Lemma 4.1. Let $M$ be any $H \mathbb{Z} / p$-module spectrum. Then $M$ is equivalent to a wedge sum of suspensions of $H \mathbb{Z} / p$. Hence $H_{*}(M)$ is a sum of shifted copies of $A_{*}$ as an $A_{*-}$ comodule, and the Hurewicz homomorphism $\pi_{*}(M) \rightarrow H_{*}(M)$ identifies $\pi_{*}(M)$ with the $A_{*}$-comodule primitives in $H_{*}(M)$. 
Proof. The module action map $\lambda: H \mathbb{Z} / p \wedge M \rightarrow M$ is a retraction, so $\pi_{*}(M)$ is a direct summand of $\pi_{*}(H \mathbb{Z} / p \wedge M)=H_{*}(M)$, hence is a graded $\mathbb{Z} / p$-vector space. Choose maps $\alpha: S^{n} \rightarrow M$ that represent a basis for this vector space. The wedge sum of the maps

$$
\lambda \circ(1 \wedge \alpha): \Sigma^{n} H \mathbb{Z} / p=H \mathbb{Z} / p \wedge S^{n} \rightarrow M
$$

is the desired $\pi_{*}$-isomorphism $\bigvee_{\alpha} \Sigma^{n} H \mathbb{Z} / p \rightarrow M$.

For each $\ell$-algebra $B, V(1) \wedge T H H(B)$ is a module spectrum over $V(1) \wedge T H H(\ell)$ and thus over $V(1) \wedge \ell \simeq H \mathbb{Z} / p$, so $H_{*}(V(1) \wedge T H H(B))$ is a sum of copies of $A_{*}$ as an $A_{*}$-comodule, by Lemma 4.1. In particular, $V(1)_{*} T H H(B)=\pi_{*}(V(1) \wedge T H H(B))$ is naturally identified with the subgroup of $A_{*}$-comodule primitives in

$$
H_{*}(V(1) \wedge T H H(B)) \cong H_{*}(V(1)) \otimes H_{*}(T H H(B))
$$

with the diagonal $A_{*}$-comodule coaction. We write $v \wedge x$ for the image of $v \otimes x$ under this identification, with $v \in H_{*}(V(1))$ and $x \in H_{*}(T H H(B))$. Let

$$
\begin{array}{rlrl}
\epsilon_{0} & =1 \wedge \bar{\tau}_{0}+\tau_{0} \wedge 1, & \mu_{0}=1 \wedge \sigma \bar{\tau}_{0}, \\
\epsilon_{1}=1 \wedge \bar{\tau}_{1}+\tau_{0} \wedge \bar{\xi}_{1}+\tau_{1} \wedge 1, & \mu_{1}=1 \wedge \sigma \bar{\tau}_{1}+\tau_{0} \wedge \sigma \bar{\xi}_{1}, \\
\lambda_{1}=1 \wedge \sigma \bar{\xi}_{1}, & & \mu_{2}=1 \wedge \sigma \bar{\tau}_{2}+\tau_{0} \wedge \sigma \bar{\xi}_{2} . \\
\lambda_{2}=1 \wedge \sigma \bar{\xi}_{2}, & &
\end{array}
$$

These are all $A_{*}$-comodule primitive, when defined, in $H_{*}(V(1) \wedge T H H(B))$ for $B=\ell$, $\ell / p, H \mathbb{Z}_{p}$ or $H \mathbb{Z} / p$ (see Remark 3.1). By a dimension count,

$$
\begin{aligned}
V(1)_{*} T H H(\mathbb{Z} / p) & =E\left(\epsilon_{0}, \epsilon_{1}\right) \otimes P\left(\mu_{0}\right), \\
V(1)_{*} T H H\left(\mathbb{Z}_{(p)}\right) & =E\left(\epsilon_{1}\right) \otimes E\left(\lambda_{1}\right) \otimes P\left(\mu_{1}\right), \\
V(1)_{*} T H H(\ell) & =E\left(\lambda_{1}, \lambda_{2}\right) \otimes P\left(\mu_{2}\right)
\end{aligned}
$$

as commutative $\mathbb{F}_{p}$-algebras. The map $\pi: \ell \rightarrow H \mathbb{Z}_{(p)}$ takes $\lambda_{2}$ to 0 and $\mu_{2}$ to $\mu_{1}^{p}$. The map $i: H \mathbb{Z}_{(p)} \rightarrow H \mathbb{Z} / p$ takes $\lambda_{1}$ to 0 and $\mu_{1}$ to $\mu_{0}^{p}$. Note that $\mu_{2} \in V(1)_{2 p^{2}} T H H(\ell)$ was simply denoted $\mu$ in [AR02].

In degrees $\leq 2 p-2$ of $H_{*}(V(1) \wedge T H H(\ell / p))$ the classes

$$
\mu_{0}^{i}:=1 \wedge\left(\sigma \bar{\tau}_{0}\right)^{i}
$$

for $0 \leq i \leq p-1$ and

$$
\epsilon_{0} \mu_{0}^{i}:=1 \wedge \bar{\tau}_{0}\left(\sigma \bar{\tau}_{0}\right)^{i}+\tau_{0} \wedge\left(\sigma \bar{\tau}_{0}\right)^{i}
$$

for $0 \leq i \leq p-2$ are $A_{*}$-comodule primitive, hence lift uniquely to $V(1)_{*} T H H(\ell / p)$. These map to the classes $\epsilon_{0}^{\delta} \mu_{0}^{i}$ in $V(1)_{*} T H H(\mathbb{Z} / p)$ for $0 \leq \delta \leq 1$ and $0 \leq i \leq p-1$, except that the degree bound excludes the top case of $\epsilon_{0} \mu_{0}^{p-1}$. 
In degree $2 p-1$ of $H_{*}(V(1) \wedge T H H(\ell / p))$ we have generators $1 \wedge \bar{\xi}_{1} \bar{\tau}_{0}, \tau_{0} \wedge\left(\sigma \bar{\tau}_{0}\right)^{p-1}$, $\tau_{0} \wedge \bar{\xi}_{1}, \tau_{1} \wedge 1$ and $1 \wedge y$. These have coactions

$$
\begin{aligned}
v\left(1 \wedge \bar{\xi}_{1} \bar{\tau}_{0}\right) & =1 \otimes 1 \wedge \bar{\xi}_{1} \bar{\tau}_{0}+\bar{\tau}_{0} \otimes 1 \wedge \bar{\xi}_{1}+\bar{\xi}_{1} \otimes 1 \wedge \bar{\tau}_{0}+\bar{\xi}_{1} \bar{\tau}_{0} \otimes 1 \wedge 1, \\
v\left(\tau_{0} \wedge\left(\sigma \bar{\tau}_{0}\right)^{p-1}\right) & =1 \otimes \tau_{0} \wedge\left(\sigma \bar{\tau}_{0}\right)^{p-1}+\tau_{0} \otimes 1 \wedge\left(\sigma \bar{\tau}_{0}\right)^{p-1}, \\
v\left(\tau_{0} \wedge \bar{\xi}_{1}\right) & =1 \otimes \tau_{0} \wedge \bar{\xi}_{1}+\tau_{0} \otimes 1 \wedge \bar{\xi}_{1}+\bar{\xi}_{1} \otimes \tau_{0} \wedge 1+\bar{\xi}_{1} \tau_{0} \otimes 1 \wedge 1, \\
v\left(\tau_{1} \wedge 1\right) & =1 \otimes \tau_{1} \wedge 1+\xi_{1} \otimes \tau_{0} \wedge 1+\tau_{1} \otimes 1 \wedge 1
\end{aligned}
$$

and

$$
v(1 \wedge y)=1 \otimes 1 \wedge y+\bar{\tau}_{0} \otimes 1 \wedge\left(\sigma \bar{\tau}_{0}\right)^{p-1}-\bar{\tau}_{0} \otimes 1 \wedge \bar{\xi}_{1}-\bar{\tau}_{1} \otimes 1 \wedge 1 .
$$

Hence the sum

$$
\bar{\epsilon}_{1}:=1 \wedge y+\tau_{0} \wedge\left(\sigma \bar{\tau}_{0}\right)^{p-1}-\tau_{0} \wedge \bar{\xi}_{1}-\tau_{1} \wedge 1
$$

is $A_{*}$-comodule primitive. Its image under $\pi_{*}$ in $H_{*}(V(1) \wedge T H H(\mathbb{Z} / p))$ is

$$
\epsilon_{0} \mu_{0}^{p-1}-\epsilon_{1}=1 \wedge \bar{\tau}_{0}\left(\sigma \bar{\tau}_{0}\right)^{p-1}+\tau_{0} \wedge\left(\sigma \bar{\tau}_{0}\right)^{p-1}-1 \wedge \bar{\tau}_{1}-\tau_{0} \wedge \bar{\xi}_{1}-\tau_{1} \wedge 1 .
$$

Let

$$
V(1)_{*} T H H(\ell) /\left(\lambda_{1}\right)=E\left(\lambda_{2}\right) \otimes P\left(\mu_{2}\right)
$$

be the quotient algebra of $V(1)_{*} T H H(\ell)$ by the ideal generated by $\lambda_{1}$.

Proposition 4.2. The classes

$$
1, \epsilon_{0}, \mu_{0}, \epsilon_{0} \mu_{0}, \ldots, \mu_{0}^{p-1}, \bar{\epsilon}_{1} \in H_{*}(V(1) \wedge T H H(\ell / p))
$$

defined in (4.2)-(4.4) have unique lifts with same names in $V(1)_{*} T H H(\ell / p)$. The graded $V(1)_{*} T H H(\ell)$-module $V(1)_{*} T H H(\ell / p)$ is a free $V(1)_{*} T H H(\ell) /\left(\lambda_{1}\right)$-module generated by these $2 p$ classes:

$$
V(1)_{*} T H H(\ell / p)=V(1)_{*} T H H(\ell) /\left(\lambda_{1}\right) \otimes \mathbb{F}_{p}\left\{1, \epsilon_{0}, \mu_{0}, \epsilon_{0} \mu_{0}, \ldots, \mu_{0}^{p-1}, \bar{\epsilon}_{1}\right\} .
$$

The map $\pi_{*}$ to $V(1)_{*} T H H(\mathbb{Z} / p)$ takes $\epsilon_{0}^{\delta} \mu_{0}^{i}$ in degree $0 \leq \delta+2 i \leq 2 p-2$ to $\epsilon_{0}^{\delta} \mu_{0}^{i}$, and takes $\bar{\epsilon}_{1}$ in degree $2 p-1$ to $\epsilon_{0} \mu_{0}^{p-1}-\epsilon_{1}$.

Proof. Additively, this follows by another dimension count, and the description of $\pi_{*}$ follows from the definition of the classes in question. It remains to prove that the action of $V(1)_{*} T H H(\ell)$ is as claimed.

The action of $\mu_{2}^{i}$ and $\lambda_{2} \mu_{2}^{i}$ in $V(1)_{*} T H H(\ell)$ on the generators

$$
1, \epsilon_{0}, \mu_{0}, \epsilon_{0} \mu_{0}, \ldots, \mu_{0}^{p-1}, \bar{\epsilon}_{1}
$$

of $V(1)_{*} T H H(\ell / p)$ is nontrivial for all $i \geq 0$, since the corresponding statement holds for the images of these classes in $H_{*}(V(1) \wedge T H H(\ell))$ and $H_{*}(V(1) \wedge T H H(\ell / p))$. This follows from Lemma 3.3 and the definition of these classes. It remains to show that $\lambda_{1}$ acts trivially on $V(1)_{*} T H H(\ell / p)$. For degree reasons, multiplication by $\lambda_{1}$ is zero on all classes except possibly $\mu_{2}^{i}$ and $\lambda_{2} \mu_{2}^{i}$, for $i \geq 0$. Because of the module structure, it suffices to show that $\lambda_{1}=\lambda_{1} \cdot 1=0$ in $V(1)_{*} T H H(\ell / p)$. This follows from the statement that the image of $\lambda_{1}$ in $H_{*}(V(1) \wedge T H H(\ell / p))$ is equal to $1 \wedge \sigma \bar{\xi}_{1}=0$, as implied by Lemma 3.3. 


\section{The $C_{p}$-Tate construction}

For the remainder of this paper, let $p$ be a prime with $p \geq 5$. We briefly recall the terminology on equivariant stable homotopy theory used below, and refer to [GM95], [HM97, $\S 1],[\mathrm{HM} 03, \S 4]$ and [AR02, §3] for more details. Let $C_{p^{n}}$ denote the cyclic group of order $p^{n}$, considered as a closed subgroup of the circle group $S^{1}$, and let $G=S^{1}$ or $C_{p^{n}}$. For each spectrum $X$ with $S^{1}$-action, let $X_{h G}=E G_{+} \wedge_{G} X$ and $X^{h G}=F\left(E G_{+}, X\right)^{G}$ denote its homotopy orbit and homotopy fixed point spectra, as usual. We now write $X^{t G}=\left[\widetilde{E G} \wedge F\left(E G_{+}, X\right)\right]^{G}$ for the $G$-Tate construction on $X$, which was denoted $t_{G}(X)^{G}$ in [GM95] and $\hat{H}(G, X)$ in [HM97], [HM03], [AR02].

We denote by $F$ the Frobenius map $X^{C^{n}} \rightarrow X^{C^{n-1}}$ given by the inclusion of fixed-point spectra, and by $V$ the Verschiebung map $X^{C_{p^{n-1}}} \rightarrow X^{C_{p^{n}}}$ given by transfer. We shall also consider the homotopy Frobenius, Tate Frobenius and homotopy Verschiebung maps $F^{h}: X^{h S^{1}} \rightarrow X^{h C_{p^{n}}}, F^{h}: X^{h C_{p^{n}}} \rightarrow X^{h C_{p^{n-1}}}, F^{t}: X^{t S^{1}} \rightarrow X^{t C_{p^{n}}}$ and $V^{h}: X^{h C_{p^{n-1}}} \rightarrow X^{h C_{p^{n}}}$.

There are conditionally convergent $G$-homotopy fixed point and $G$-Tate spectral sequences in $V(1)$-homotopy for $X$, with

$$
E_{s, t}^{2}(G, X)=H_{\mathrm{gp}}^{-s}\left(G ; V(1)_{t}(X)\right) \Rightarrow V(1)_{s+t}\left(X^{h G}\right)
$$

and

$$
\hat{E}_{s, t}^{2}(G, X)=\hat{H}_{\mathrm{gp}}^{-s}\left(G ; V(1)_{t}(X)\right) \Rightarrow V(1)_{s+t}\left(X^{t G}\right) .
$$

Here $H_{\mathrm{gp}}^{*}\left(G ; V(1)_{*}(X)\right)$ denotes the group cohomology of $G$ and $\hat{H}_{\mathrm{gp}}^{*}\left(G ; V(1)_{*}(X)\right)$ the Tate cohomology of $G$, with coefficients in $V(1)_{*}(X)$. Notice that in our case, with $X=$ $T H H(B)$, the action of $G$ on $V(1)_{*}(X)$ is trivial, since it is the restriction of an $S^{1}$-action. We write $H_{\mathrm{gp}}^{*}\left(C_{p^{n}} ; \mathbb{F}_{p}\right)=E\left(u_{n}\right) \otimes P(t)$ and $\hat{H}_{\mathrm{gp}}^{*}\left(C_{p^{n}} ; \mathbb{F}_{p}\right)=E\left(u_{n}\right) \otimes P\left(t^{ \pm 1}\right)$ with $u_{n}$ in degree 1 and $t$ in degree 2 (see for example [Ben98, Prop. 3.5.5] and [HM03, Lem. 4.2.1]). So $u_{n}, t$ and $x \in V(1)_{t}(X)$ have bidegree $(-1,0),(-2,0)$ and $(0, t)$ in either spectral sequence, respectively. See [HM03, §4.3] for proofs of the multiplicative properties of these spectral sequences. Similarly, we write $H_{\mathrm{gp}}^{*}\left(S^{1} ; \mathbb{F}_{p}\right)=P(t)$ and $\hat{H}_{\mathrm{gp}}^{*}\left(S^{1} ; \mathbb{F}_{p}\right)=$ $P\left(t^{ \pm 1}\right)$. We have morphisms of spectral sequences induced by the homotopy and Tate Frobenii, which on the $E^{2}$-terms map $t$ to $t$ and $u_{n}$ to zero.

We are principally interested in the case when $X=T H H(B)$, with the $S^{1}$-action given by the cyclic structure [Lod98, Def. 7.1.9], [HM03, §1.2]. It is a cyclotomic spectrum, in the sense of [HM97, §1], leading to the commutative diagram

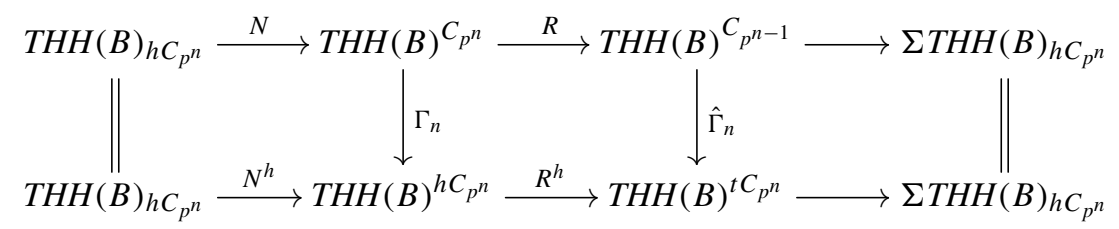

of horizontal cofibre sequences. We abbreviate $\hat{E}^{2}(G, T H H(B))$ to $\hat{E}^{2}(G, B)$, etc. When $B$ is a commutative $S$-algebra, this is a commutative algebra spectral sequence, and 
when $B$ is an associative $A$-algebra, with $A$ commutative, then $\hat{E}^{*}(G, B)$ is a module spectral sequence over $\hat{E}^{*}(G, A)$. The map $R^{h}$ corresponds to the inclusion $E^{2}(G, B) \rightarrow$ $\hat{E}^{2}(G, B)$ from the second quadrant to the upper half-plane, for connective $B$.

Definition 5.1. We call a homomorphism of graded groups $k$-coconnected if it is an isomorphism in all dimensions greater than $k$ and injective in dimension $k$.

In this section we compute $V(1)_{*} T H H(\ell / p)^{t} C_{p}$ by means of the $C_{p}$-Tate spectral sequence in $V(1)$-homotopy for $T H H(\ell / p)$. In Propositions 5.7 and 5.8 we show that the comparison map $\hat{\Gamma}_{1}: V(1)_{*} T H H(\ell / p) \rightarrow V(1)_{*} T H H(\ell / p)^{t C_{p}}$ is $(2 p-2)$-coconnected and can be identified with the algebraic localization homomorphism that inverts $\mu_{2}$.

First we recall the structure of the $C_{p}$-Tate spectral sequence for $T H H(\mathbb{Z} / p)$, with $V(0)$ - and $V(1)$-coefficients. We have $V(0)_{*} T H H(\mathbb{Z} / p)=E\left(\epsilon_{0}\right) \otimes P\left(\mu_{0}\right)$, and (with an obvious notation for the case of $V(0)$-homotopy) the $E^{2}$-terms are

$$
\begin{aligned}
\hat{E}^{2}\left(C_{p}, \mathbb{Z} / p ; V(0)\right) & =E\left(u_{1}\right) \otimes P\left(t^{ \pm 1}\right) \otimes E\left(\epsilon_{0}\right) \otimes P\left(\mu_{0}\right), \\
\hat{E}^{2}\left(C_{p}, \mathbb{Z} / p\right) & =E\left(u_{1}\right) \otimes P\left(t^{ \pm 1}\right) \otimes E\left(\epsilon_{0}, \epsilon_{1}\right) \otimes P\left(\mu_{0}\right) .
\end{aligned}
$$

In each $G$-Tate spectral sequence we have a first differential

$$
d^{2}(x)=t \cdot \sigma x
$$

(see e.g. [Rog98, §3.3]). We easily deduce $\sigma \epsilon_{0}=\mu_{0}$ and $\sigma \epsilon_{1}=\mu_{0}^{p}$ from (4.1), so

$$
\begin{aligned}
\hat{E}^{3}\left(C_{p}, \mathbb{Z} / p ; V(0)\right) & =E\left(u_{1}\right) \otimes P\left(t^{ \pm 1}\right), \\
\hat{E}^{3}\left(C_{p}, \mathbb{Z} / p\right) & =E\left(u_{1}\right) \otimes P\left(t^{ \pm 1}\right) \otimes E\left(\epsilon_{0} \mu_{0}^{p-1}-\epsilon_{1}\right) .
\end{aligned}
$$

Thus the $V(0)$-homotopy spectral sequence collapses at $\hat{E}^{3}=\hat{E}^{\infty}$. By naturality with respect to the map $i_{1}: V(0) \rightarrow V(1)$, all the classes on the horizontal axis of $\hat{E}^{3}\left(C_{p}, \mathbb{Z} / p\right)$ are infinite cycles, so also the latter spectral sequence collapses at $\hat{E}^{3}\left(C_{p}, \mathbb{Z} / p\right)$.

We know from [HM03, Cor. 4.4.2] that the comparison map

$$
\hat{\Gamma}_{1}: V(0)_{*} T H H(\mathbb{Z} / p) \rightarrow V(0)_{*} T H H(\mathbb{Z} / p)^{t C_{p}}
$$

takes $\epsilon_{0}^{\delta} \mu_{0}^{i}$ to $\left(u_{1} t^{-1}\right)^{\delta} t^{-i}$ for all $0 \leq \delta \leq 1, i \geq 0$. In particular, the integral map $\hat{\Gamma}_{1}: \pi_{*} T H H(\mathbb{Z} / p) \rightarrow \pi_{*} T H H(\mathbb{Z} / p)^{t C_{p}}$ is $(-2)$-coconnected. From this we can deduce the following behaviour of the comparison map $\hat{\Gamma}_{1}$ in $V(1)$-homotopy.

Lemma 5.2. The map

$$
\hat{\Gamma}_{1}: V(1)_{*} T H H(\mathbb{Z} / p) \rightarrow V(1)_{*} T H H(\mathbb{Z} / p)^{t C_{p}}
$$

takes the classes $\epsilon_{0}^{\delta} \mu_{0}^{i}$ from $V(0)_{*} T H H(\mathbb{Z} / p)$, for $0 \leq \delta \leq 1$ and $i \geq 0$, to classes represented in $\hat{E}^{\infty}\left(C_{p}, \mathbb{Z} / p\right)$ by $\left(u_{1} t^{-1}\right)^{\delta} t^{-i}$ (on the horizontal axis). Furthermore, it takes the class $\epsilon_{0} \mu_{0}^{p-1}-\epsilon_{1}$ in degree $2 p-1$ to a class represented by $\epsilon_{0} \mu_{0}^{p-1}-\epsilon_{1}$ (on the vertical axis). 
Proof. The classes $\epsilon_{0}^{\delta} \mu_{0}^{i}$ are in the image from $V(0)$-homotopy, and we recalled above that they are detected by $\left(u_{1} t^{-1}\right)^{\delta} t^{-i}$ in the $V(0)$-homotopy $C_{p}$-Tate spectral sequence for $\operatorname{THH}(\mathbb{Z} / p)$. By naturality along $i_{1}: V(0) \rightarrow V(1)$, they are detected by the same (nonzero) classes in the $V(1)$-homotopy spectral sequence $\hat{E}^{\infty}\left(C_{p}, \mathbb{Z} / p\right)$.

To find the representative for $\hat{\Gamma}_{1}\left(\epsilon_{0} \mu_{0}^{p-1}-\epsilon_{1}\right)$ in degree $2 p-1$, we appeal to the cyclotomic trace map from algebraic $K$-theory, or more precisely, to the commutative diagram

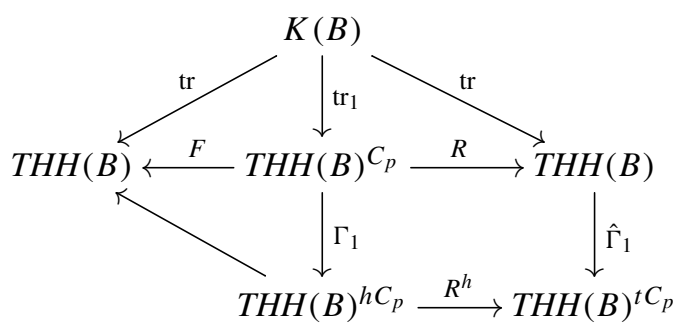

The Bökstedt trace map tr: $K(B) \rightarrow T H H(B)$ admits a preferred lift $\operatorname{tr}_{n}$ through each fixed point spectrum $T H H(B){ }^{C_{p^{n}}}$, which homotopy equalizes the iterated restriction and Frobenius maps $R^{n}$ and $F^{n}$ to $T H H(B)$ (see [Dun04, §3]). In particular, the $\sigma$-operator on $V(1)_{*} T H H(B)$ is zero on classes in the image of tr.

In the case $B=H \mathbb{Z} / p$ we know that $K(\mathbb{Z} / p)_{p} \simeq H \mathbb{Z}_{p}$, so $V(1)_{*} K(\mathbb{Z} / p)=E\left(\bar{\epsilon}_{1}\right)$, where the $v_{1}$-Bockstein of $\bar{\epsilon}_{1}$ is -1 . The Bökstedt trace image $\operatorname{tr}\left(\bar{\epsilon}_{1}\right) \in V(1)_{*} T H H(\mathbb{Z} / p)$ lies in $\mathbb{F}_{p}\left\{\epsilon_{1}, \epsilon_{0} \mu_{0}^{p-1}\right\}$, has $v_{1}$-Bockstein $\operatorname{tr}(-1)=-1$ and suspends by $\sigma$ to 0 . Hence

$$
\operatorname{tr}\left(\bar{\epsilon}_{1}\right)=\epsilon_{0} \mu_{0}^{p-1}-\epsilon_{1}
$$

As we recalled above, the map $\hat{\Gamma}_{1}: \pi_{*} T H H(\mathbb{Z} / p) \rightarrow \pi_{*} T H H(\mathbb{Z} / p)^{t C_{p}}$ is $(-2)$-coconnected, so the corresponding map in $V(1)$-homotopy is at least $(2 p-2)$-coconnected. Thus it takes $\epsilon_{0} \mu_{0}^{p-1}-\epsilon_{1}$ to a nonzero class in $V(1)_{*} T H H(\mathbb{Z} / p)^{t C_{p}}$, represented somewhere in total degree $2 p-1$ of $\hat{E}^{\infty}\left(C_{p}, \mathbb{Z} / p\right)$, in the lower right hand corner of the diagram.

Going down the middle part of the diagram, we reach a class $\left(\Gamma_{1} \circ \operatorname{tr}_{1}\right)\left(\bar{\epsilon}_{1}\right)$, represented in total degree $(2 p-1)$ in the left half-plane $C_{p}$-homotopy fixed point spectral sequence $E^{\infty}\left(C_{p}, \mathbb{Z} / p\right)$. Its image under the edge homomorphism to $V(1)_{*} T H H(\mathbb{Z} / p)$ equals $\left(F \circ \operatorname{tr}_{1}\right)\left(\bar{\epsilon}_{1}\right)=\operatorname{tr}\left(\bar{\epsilon}_{1}\right)$, hence $\left(\Gamma_{1} \circ \operatorname{tr}_{1}\right)\left(\bar{\epsilon}_{1}\right)$ is represented by $\epsilon_{0} \mu_{0}^{p-1}-\epsilon_{1}$ in $E_{0,2 p-1}^{\infty}\left(C_{p}, \mathbb{Z} / p\right)$. Its image under $R^{h}$ in the $C_{p}$-Tate spectral sequence is the generator of $\hat{E}_{0,2 p-1}^{\infty}\left(C_{p}, \mathbb{Z} / p\right)=\mathbb{F}_{p}\left\{\epsilon_{0} \mu_{0}^{p-1}-\epsilon_{1}\right\}$, hence that generator is the $E^{\infty}$-representative of $\hat{\Gamma}_{1}\left(\epsilon_{0} \mu_{0}^{p-1}-\epsilon_{1}\right)$.

The $(2 p-2)$-connected map $\pi: \ell / p \rightarrow H \mathbb{Z} / p$ induces a $(2 p-1)$-connected map $V(1)_{*} K(\ell / p) \rightarrow V(1)_{*} K(\mathbb{Z} / p)=E\left(\bar{\epsilon}_{1}\right)$, by [BM94, Prop. 10.9]. We can lift the algebraic $K$-theory class $\bar{\epsilon}_{1}$ to $\ell / p$. This lift is not unique, but we fix one choice. 
Definition 5.3. We call

$$
\bar{\epsilon}_{1}^{K} \in V(1)_{2 p-1} K(\ell / p)
$$

a chosen class that maps to the generator $\bar{\epsilon}_{1}$ in $V(1)_{2 p-1} K(\mathbb{Z} / p) \cong \mathbb{Z} / p$.

Lemma 5.4. The Bökstedt trace $\operatorname{tr}: V(1)_{*} K(\ell / p) \rightarrow V(1)_{*} T H H(\ell / p)$ takes $\bar{\epsilon}_{1}^{K}$ to $\bar{\epsilon}_{1}$.

Proof. In the commutative square

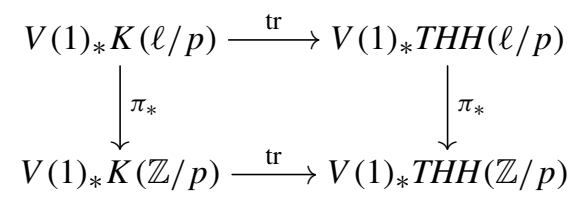

the trace image $\operatorname{tr}\left(\bar{\epsilon}_{1}^{K}\right)$ in $V(1)_{*} T H H(\ell / p)$ must map under $\pi_{*} \operatorname{to} \operatorname{tr}\left(\bar{\epsilon}_{1}\right)=\epsilon_{0} \mu_{0}^{p-1}-\epsilon_{1}$ in $V(1)_{*} T H H(\mathbb{Z} / p)$, which by Proposition 4.2 characterizes it as being equal to the class $\bar{\epsilon}_{1}$. Hence $\operatorname{tr}\left(\bar{\epsilon}_{1}^{K}\right)=\bar{\epsilon}_{1}$.

Next we turn to the $C_{p}$-Tate spectral sequence $\hat{E}^{*}\left(C_{p}, \ell / p\right)$ in $V(1)$-homotopy for $T H H(\ell / p)$. Its $E^{2}$-term is

$\hat{E}^{2}\left(C_{p}, \ell / p\right)=E\left(u_{1}\right) \otimes P\left(t^{ \pm 1}\right) \otimes \mathbb{F}_{p}\left\{1, \epsilon_{0}, \mu_{0}, \epsilon_{0} \mu_{0}, \ldots, \mu_{0}^{p-1}, \bar{\epsilon}_{1}\right\} \otimes E\left(\lambda_{2}\right) \otimes P\left(\mu_{2}\right)$.

We have $d^{2}(x)=t \cdot \sigma x$, where

$$
\sigma\left(\epsilon_{0}^{\delta} \mu_{0}^{i-1}\right)= \begin{cases}\mu_{0}^{i} & \text { for } \delta=1,0<i<p \\ 0 & \text { otherwise }\end{cases}
$$

is readily deduced from (4.1), and $\sigma\left(\bar{\epsilon}_{1}\right)=0$ since $\bar{\epsilon}_{1}$ is in the image of tr. Thus

$$
\hat{E}^{3}\left(C_{p}, \ell / p\right)=E\left(u_{1}\right) \otimes P\left(t^{ \pm 1}\right) \otimes E\left(\bar{\epsilon}_{1}\right) \otimes E\left(\lambda_{2}\right) \otimes P\left(t \mu_{2}\right) .
$$

We prefer to use $t \mu_{2}$ rather than $\mu_{2}$ as a generator, since it represents multiplication by $v_{2}$ (up to a unit factor in $\mathbb{F}_{p}$ ) in all module spectral sequences over $E^{*}\left(S^{1}, \ell\right.$ ), by [AR02, Prop. 4.8].

To proceed, we shall use that $\hat{E}^{*}\left(C_{p}, \ell / p\right)$ is a module over the spectral sequence for $\operatorname{THH}(\ell)$. We therefore recall the structure of the latter spectral sequence, from [AR02, Th. 5.5]. It begins

$$
\hat{E}^{2}\left(C_{p}, \ell\right)=E\left(u_{1}\right) \otimes P\left(t^{ \pm 1}\right) \otimes E\left(\lambda_{1}, \lambda_{2}\right) \otimes P\left(\mu_{2}\right) .
$$

The classes $\lambda_{1}, \lambda_{2}$ and $t \mu_{2}$ are infinite cycles, and the differentials

$$
d^{2 p}\left(t^{1-p}\right) \doteq t \lambda_{1}, \quad d^{2 p^{2}}\left(t^{p-p^{2}}\right) \doteq t^{p} \lambda_{2}, \quad d^{2 p^{2}+1}\left(u_{1} t^{-p^{2}}\right) \doteq t \mu_{2}
$$

leave the terms

$$
\hat{E}^{2 p+1}\left(C_{p}, \ell\right)=E\left(u_{1}, \lambda_{1}, \lambda_{2}\right) \otimes P\left(t^{ \pm p}, t \mu_{2}\right),
$$




$$
\begin{aligned}
& \hat{E}^{2 p^{2}+1}\left(C_{p}, \ell\right)=E\left(u_{1}, \lambda_{1}, \lambda_{2}\right) \otimes P\left(t^{ \pm p^{2}}, t \mu_{2}\right), \\
& \hat{E}^{2 p^{2}+2}\left(C_{p}, \ell\right)=E\left(\lambda_{1}, \lambda_{2}\right) \otimes P\left(t^{ \pm p^{2}}\right),
\end{aligned}
$$

with $\hat{E}^{2 p^{2}+2}=\hat{E}^{\infty}$, converging to $V(1)_{*} T H H(\ell)^{t C_{p}}$. The comparison map $\hat{\Gamma}_{1}$ takes $\lambda_{1}, \lambda_{2}, \mu_{2}$ to $\lambda_{1}, \lambda_{2}, t^{-p^{2}}$ (up to a unit factor in $\mathbb{F}_{p}$ ), respectively, inducing the algebraic localization map and identification

$$
\hat{\Gamma}_{1}: V(1)_{*} T H H(\ell) \rightarrow V(1)_{*} T H H(\ell)\left[\mu_{2}^{-1}\right] \cong V(1)_{*} T H H(\ell)^{t C_{p}} .
$$

Lemma 5.5. In $\hat{E}^{*}\left(C_{p}, \ell / p\right)$, the class $u_{1} t^{-p}$ supports the nonzero differential

$$
d^{2 p^{2}}\left(u_{1} t^{-p}\right) \doteq u_{1} t^{p^{2}-p} \lambda_{2},
$$

and does not survive to the $E^{\infty}$-term.

Proof. In $\hat{E}^{*}\left(C_{p}, \ell\right)$, there is such a differential. By naturality along $i: \ell \rightarrow \ell / p$, it follows that there is also such a differential in $\hat{E}^{*}\left(C_{p}, \ell / p\right)$. It remains to argue that the target class is nonzero at the $E^{2 p^{2}}$-term. Considering the $E^{3}$-term in (5.2), the only possible source of a previous differential hitting $u_{1} t^{p^{2}-p} \lambda_{2}$ is $\bar{\epsilon}_{1}$, supporting a $d^{2 p^{2}-2 p+1}$ differential. But $\bar{\epsilon}_{1}$ is in an even column and $u_{1} t^{p^{2}-p} \lambda_{2}$ is in an odd column. By naturality with respect to the Tate Frobenius map $F^{t}: T H H(\ell / p)^{t S^{1}} \rightarrow T H H(\ell / p)^{t C_{p}}$, any such differential from an even to an odd column must be zero. Indeed, the $S^{1}$-Tate spectral sequence has $E^{2}$-term given by $P\left(t^{ \pm 1}\right) \otimes V(1)_{*} T H H(\ell / p)$, and $F^{t}$ induces the injective homomorphism that takes $\hat{E}^{2}\left(S^{1}, \ell / p\right)$ isomorphically to the even columns of $\hat{E}^{2}\left(C_{p}, \ell / p\right)$. Since $\hat{E}^{*}\left(S^{1}, \ell / p\right)$ is concentrated in even columns, all differentials of odd length are zero. By naturality, classes of $\hat{E}^{r}\left(C_{p}, \ell / p\right)$ that lie in the image of $\hat{E}^{r}\left(F^{t}\right)$ cannot support a differential of odd length (cf. [AR02, Lem. 5.2]). In the present situation, the $d^{2}$-differential of $\hat{E}^{*}\left(C_{p}, \ell / p\right)$ leading to (5.2) is also nonzero in $\hat{E}^{*}\left(S^{1}, \ell / p\right)$, so that

$$
\hat{E}^{3}\left(S^{1}, \ell / p\right)=P\left(t^{ \pm 1}\right) \otimes E\left(\bar{\epsilon}_{1}\right) \otimes E\left(\lambda_{2}\right) \otimes P\left(t \mu_{2}\right) .
$$

By inspection, if the class $\bar{\epsilon}_{1} \in \hat{E}^{2}\left(C_{p}, \ell / p\right)$ survives to $\hat{E}^{2 p^{2}-2 p+1}\left(C_{p}, \ell / p\right)$, then it will lie in the image of $\hat{E}^{2 p^{2}-2 p+1}\left(F^{t}\right)$.

To determine the map $\hat{\Gamma}_{1}$ we use naturality with respect to the map $\pi: \ell / p \rightarrow H \mathbb{Z} / p$.

Lemma 5.6. The classes $1, \epsilon_{0}, \mu_{0}, \epsilon_{0} \mu_{0}, \ldots, \mu_{0}^{p-1}$ and $\bar{\epsilon}_{1}$ in $V(1)_{*} T H H(\ell / p)$ map under $\hat{\Gamma}_{1}$ to classes in $V(1)_{*} T H H(\ell / p)^{t C_{p}}$ that are represented in $\hat{E}^{\infty}\left(C_{p}, \ell / p\right)$ by the permanent cycles $\left(u_{1} t^{-1}\right)^{\delta} t^{-i}$ (on the horizontal axis) in degrees $\leq 2 p-2$, and by the permanent cycle $\bar{\epsilon}_{1}$ (on the vertical axis) in degree $2 p-1$.

Proof. In the commutative square

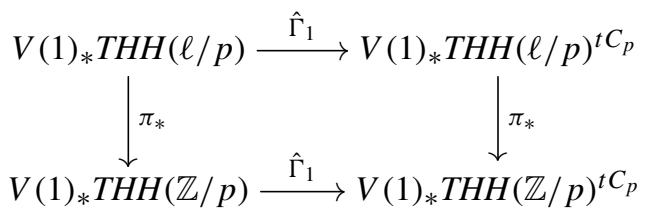


the classes $\epsilon_{0}^{\delta} \mu_{0}^{i}$ in the upper left corner map to classes in the lower right corner that are represented by $\left(u_{1} t^{-1}\right)^{\delta} t^{-i}$ in degrees $\leq 2 p-2$, and $\bar{\epsilon}_{1}$ maps to $\epsilon_{0} \mu_{0}^{p-1}-\epsilon_{1}$ in degree $2 p-1$. This follows by combining Proposition 4.2 and Lemma 5.2.

The first $2 p-1$ of these are represented in maximal filtration (on the horizontal axis), so their images in the upper right corner must be represented by permanent cycles $\left(u_{1} t^{-1}\right)^{\delta} t^{-i}$ in the Tate spectral sequence $\hat{E}^{\infty}\left(C_{p}, \ell / p\right)$.

The image of the last class, $\bar{\epsilon}_{1}$, in the upper right corner could either be represented by $\bar{\epsilon}_{1}$ in bidegree $(0,2 p-1)$ or by $u_{1} t^{-p}$ in bidegree $(2 p-1,0)$. However, the last class supports a differential $d^{2 p^{2}}\left(u_{1} t^{-p}\right) \doteq u_{1} t^{p^{2}-p} \lambda_{2}$, by Lemma 5.5 above. This only leaves the other possibility, that $\hat{\Gamma}_{1}\left(\bar{\epsilon}_{1}\right)$ is represented by $\bar{\epsilon}_{1}$ in $\hat{E}^{\infty}\left(C_{p}, \ell / p\right)$.

We proceed to determine the differential structure in $\hat{E}^{*}\left(C_{p}, \ell / p\right)$, making use of the permanent cycles identified above.

Proposition 5.7. The $C_{p}$-Tate spectral sequence in $V(1)$-homotopy for $T H H(\ell / p)$ has

$$
\hat{E}^{3}\left(C_{p}, \ell / p\right)=E\left(u_{1}, \bar{\epsilon}_{1}, \lambda_{2}\right) \otimes P\left(t^{ \pm 1}, t \mu_{2}\right) .
$$

It has differentials generated by

$$
\begin{aligned}
& d^{2 p^{2}-2 p+2}\left(t^{p-p^{2}} \cdot t^{-i} \bar{\epsilon}_{1}\right) \doteq t \mu_{2} \cdot t^{-i} \\
& \text { for } 0<i<p, d^{2 p^{2}}\left(t^{p-p^{2}}\right) \doteq t^{p} \lambda_{2} \text { and } d^{2 p^{2}+1}\left(u_{1} t^{-p^{2}}\right) \doteq t \mu_{2} . \text { The subsequent terms } \\
& \qquad \begin{aligned}
\hat{E}^{2 p^{2}-2 p+3}\left(C_{p}, \ell / p\right)= & E\left(u_{1}, \lambda_{2}\right) \otimes \mathbb{F}_{p}\left\{t^{-i} \mid 0<i<p\right\} \otimes P\left(t^{ \pm p}\right) \\
& \oplus E\left(u_{1}, \bar{\epsilon}_{1}, \lambda_{2}\right) \otimes P\left(t^{ \pm p}, t \mu_{2}\right), \\
\hat{E}^{2 p^{2}+1}\left(C_{p}, \ell / p\right)= & E\left(u_{1}, \lambda_{2}\right) \otimes \mathbb{F}_{p}\left\{t^{-i} \mid 0<i<p\right\} \otimes P\left(t^{ \pm p^{2}}\right) \\
& \oplus E\left(u_{1}, \bar{\epsilon}_{1}, \lambda_{2}\right) \otimes P\left(t^{ \pm p^{2}}, t \mu_{2}\right), \\
\hat{E}^{2 p^{2}+2}\left(C_{p}, \ell / p\right)= & E\left(u_{1}, \lambda_{2}\right) \otimes \mathbb{F}_{p}\left\{t^{-i} \mid 0<i<p\right\} \otimes P\left(t^{ \pm p^{2}}\right) \\
& \oplus E\left(\bar{\epsilon}_{1}, \lambda_{2}\right) \otimes P\left(t^{ \pm p^{2}}\right) .
\end{aligned}
\end{aligned}
$$

The last term can be rewritten as

$$
\hat{E}^{\infty}\left(C_{p}, \ell / p\right)=\left(E\left(u_{1}\right) \otimes \mathbb{F}_{p}\left\{t^{-i} \mid 0<i<p\right\} \oplus E\left(\bar{\epsilon}_{1}\right)\right) \otimes E\left(\lambda_{2}\right) \otimes P\left(t^{ \pm p^{2}}\right) .
$$

Proof. We have already identified the $E^{2}$ - and $E^{3}$-terms above. The $E^{3}$-term (5.2) is generated over $\hat{E}^{3}\left(C_{p}, \ell\right)$ by an $\mathbb{F}_{p}$-basis for $E\left(\bar{\epsilon}_{1}\right)$, so the next possible differential is induced by $d^{2 p}\left(t^{1-p}\right) \doteq t \lambda_{1}$. But multiplication by $\lambda_{1}$ is trivial in $V(1)_{*} T H H(\ell / p)$, by Proposition 4.2 , so $\hat{E}^{3}\left(C_{p}, \ell / p\right)=\hat{E}^{2 p+1}\left(C_{p}, \ell / p\right)$. This term is generated over $\hat{E}^{2 p+1}\left(C_{p}, \ell\right)$ by $P_{p}\left(t^{-1}\right) \otimes E\left(\bar{\epsilon}_{1}\right)$. Here $1, t^{-1}, \ldots, t^{1-p}$ and $\bar{\epsilon}_{1}$ are permanent cycles, by Lemma 5.6. Any $d^{r}$-differential before $d^{2 p^{2}}$ must therefore originate on a class $t^{-i} \bar{\epsilon}_{1}$ for $0<i<p$, and be of even length $r$, since these classes lie in even columns. For bidegree reasons, the first possibility is $r=2 p^{2}-2 p+2$, so $\hat{E}^{3}\left(C_{p}, \ell / p\right)=\hat{E}^{2 p^{2}-2 p+2}\left(C_{p}, \ell / p\right)$. 
Multiplication by $v_{2}$ acts trivially on $V(1)_{*} T H H(\ell)$ and $V(1)_{*} T H H(\ell)^{t C_{p}}$ for degree reasons, and therefore also on $V(1)_{*} T H H(\ell / p)$ and $V(1)_{*} T H H(\ell / p)^{t C_{p}}$ by the module structure. The class $v_{2}$ maps to $t \mu_{2}$ in the $S^{1}$-Tate spectral sequence for $\ell$, as recalled above, so multiplication by $v_{2}$ is represented by multiplication by $t \mu_{2}$ in the $C_{p}$-Tate spectral sequence for $\ell / p$. Applied to the permanent cycles $\left(u_{1} t^{-1}\right)^{\delta} t^{-i}$ in degrees $\leq$ $2 p-2$, this implies that the products

$$
t \mu_{2} \cdot\left(u_{1} t^{-1}\right)^{\delta} t^{-i}
$$

must be infinite cycles representing zero, i.e., they must be hit by differentials. In the cases $\delta=1,0 \leq i \leq p-2$, these classes in odd columns cannot be hit by differentials of odd length, such as $d^{2 p^{2}+1}$, so the only possibility is

$$
d^{2 p^{2}-2 p+2}\left(t^{p-p^{2}} \cdot\left(u_{1} t^{-1}\right) t^{-i} \bar{\epsilon}_{1}\right) \doteq t \mu_{2} \cdot\left(u_{1} t^{-1}\right) t^{-i}
$$

for $0 \leq i \leq p-2$. By the module structure (consider multiplication by $u_{1}$ ) it follows that

$$
d^{2 p^{2}-2 p+2}\left(t^{p-p^{2}} \cdot t^{-i} \bar{\epsilon}_{1}\right) \doteq t \mu_{2} \cdot t^{-i}
$$

for $0<i<p$. Hence we can compute from (5.2) that

$$
\begin{aligned}
\hat{E}^{2 p^{2}-2 p+3}\left(C_{p}, \ell / p\right)= & E\left(u_{1}\right) \otimes P\left(t^{ \pm p}\right) \otimes \mathbb{F}_{p}\left\{t^{-i} \mid 0<i<p\right\} \otimes E\left(\lambda_{2}\right) \\
& \oplus E\left(u_{1}\right) \otimes P\left(t^{ \pm p}\right) \otimes E\left(\bar{\epsilon}_{1}\right) \otimes E\left(\lambda_{2}\right) \otimes P\left(t \mu_{2}\right)
\end{aligned}
$$

This is generated over $\hat{E}^{2 p+1}\left(C_{p}, \ell\right)$ by the permanent cycles $1, t^{-1}, \ldots, t^{1-p}$ and $\bar{\epsilon}_{1}$, so the next differential is induced by $d^{2 p^{2}}\left(t^{p-p^{2}}\right) \doteq t^{p} \lambda_{2}$. This leaves

$$
\begin{aligned}
\hat{E}^{2 p^{2}+1}\left(C_{p}, \ell / p\right)= & E\left(u_{1}\right) \otimes P\left(t^{ \pm p^{2}}\right) \otimes \mathbb{F}_{p}\left\{t^{-i} \mid 0<i<p\right\} \otimes E\left(\lambda_{2}\right) \\
& \oplus E\left(u_{1}\right) \otimes P\left(t^{ \pm p^{2}}\right) \otimes E\left(\bar{\epsilon}_{1}\right) \otimes E\left(\lambda_{2}\right) \otimes P\left(t \mu_{2}\right) .
\end{aligned}
$$

Finally, $d^{2 p^{2}+1}\left(u_{1} t^{-p^{2}}\right) \doteq t \mu_{2}$ applies, and leaves

$$
\begin{aligned}
\hat{E}^{2 p^{2}+2}\left(C_{p}, \ell / p\right)= & E\left(u_{1}\right) \otimes P\left(t^{ \pm p^{2}}\right) \otimes \mathbb{F}_{p}\left\{t^{-i} \mid 0<i<p\right\} \otimes E\left(\lambda_{2}\right) \\
& \oplus P\left(t^{ \pm p^{2}}\right) \otimes E\left(\bar{\epsilon}_{1}\right) \otimes E\left(\lambda_{2}\right) .
\end{aligned}
$$

For bidegree reasons, $\hat{E}^{2 p^{2}+2}=\hat{E}^{\infty}$.

Proposition 5.8. The comparison map $\hat{\Gamma}_{1}$ takes the classes

$$
\epsilon_{0}^{\delta} \mu_{0}^{i}, \bar{\epsilon}_{1}, \lambda_{2} \text { and } \mu_{2} \quad \text { in } V(1)_{*} T H H(\ell / p)
$$

to classes in $V(1)_{*} T H H(\ell / p)^{t C_{p}}$ represented by

$$
\left(u_{1} t^{-1}\right)^{\delta} t^{-i}, \bar{\epsilon}_{1}, \lambda_{2} \text { and } t^{-p^{2}} \quad \text { in } \hat{E}^{\infty}\left(C_{p}, \ell / p\right),
$$


up to a unit factor in $\mathbb{F}_{p}$, respectively. Thus

$$
V(1)_{*} T H H(\ell / p)^{t C_{p}} \cong \mathbb{F}_{p}\left\{1, \epsilon_{0}, \mu_{0}, \epsilon_{0} \mu_{0}, \ldots, \mu_{0}^{p-1}, \bar{\epsilon}_{1}\right\} \otimes E\left(\lambda_{2}\right) \otimes P\left(\mu_{2}^{ \pm 1}\right)
$$

and $\hat{\Gamma}_{1}$ induces an identification $V(1)_{*} T H H(\ell / p)\left[\mu_{2}^{-1}\right] \cong V(1)_{*} T H H(\ell / p)^{t C_{p}}$. In particular, $\hat{\Gamma}_{1}$ factors as the algebraic localization map and identification

$$
\hat{\Gamma}_{1}: V(1)_{*} T H H(\ell / p) \rightarrow V(1)_{*} T H H(\ell / p)\left[\mu_{2}^{-1}\right] \cong V(1)_{*} T H H(\ell / p)^{t C_{p}},
$$

and is $(2 p-2)$-coconnected.

Proof. The image under $\hat{\Gamma}_{1}$ of the classes $1, \epsilon_{0}, \mu_{0}, \epsilon_{0} \mu_{0}, \ldots, \mu_{0}^{p-1}$ and $\bar{\epsilon}_{1}$ was given in Lemma 5.6, and the action on the classes $\lambda_{2}$ and $\mu_{2}$ is given in the proof of [AR02, Th. 5.5]. The structure of $V(1)_{*} T H H(\ell / p)^{t C_{p}}$ is then immediate from the $E^{\infty}$-term in Proposition 5.7. The top class not in the image of $\hat{\Gamma}_{1}$ is $\bar{\epsilon}_{1} \lambda_{2} \mu_{2}^{-1}$, in degree $2 p-2$.

Recall that

$$
T F(B ; p)=\underset{n, F}{\operatorname{holim}} \operatorname{TH} H(B)^{C_{p^{n}}}, \quad T R(B ; p)=\underset{n, R}{\operatorname{holim}} \operatorname{THH}(B)^{C_{p^{n}}}
$$

are defined as the homotopy limits over the Frobenius and the restriction maps

$$
F, R: T H H(B)^{C_{p^{n}}} \rightarrow T H H(B)^{C_{p^{n-1}},}
$$

respectively.

Corollary 5.9. The comparison maps

$$
\begin{aligned}
& \Gamma_{n}: T H H(\ell / p)^{C_{p^{n}}} \rightarrow \operatorname{THH}(\ell / p)^{h C_{p^{n}}}, \\
& \hat{\Gamma}_{n}: T H H(\ell / p)^{C_{p^{n-1}}} \rightarrow T H H(\ell / p)^{t C_{p^{n}}}
\end{aligned}
$$

for $n \geq 1$, and

$$
\begin{aligned}
& \Gamma: T F(\ell / p ; p) \rightarrow T H H(\ell / p)^{h S^{1}}, \\
& \hat{\Gamma}: T F(\ell / p ; p) \rightarrow T H H(\ell / p)^{t S^{1}}
\end{aligned}
$$

all induce $(2 p-2)$-coconnected homomorphisms on $V(1)$-homotopy.

Proof. This follows from a theorem of Tsalidis [Tsa98, Th. 2.4] and Proposition 5.8 above, just as in [AR02, Th. 5.7]. See also [BBLNR, Ex. 10.2].

\section{Higher fixed points}

Let $n \geq 1$. Write $v_{p}(i)$ for the $p$-adic valuation of $i$. Define a numerical function $\rho(-)$ by

$$
\begin{aligned}
\rho(2 k-1) & =\left(p^{2 k+1}+1\right) /(p+1)=p^{2 k}-p^{2 k-1}+\cdots-p+1, \\
\rho(2 k) & =\left(p^{2 k+2}-p^{2}\right) /\left(p^{2}-1\right)=p^{2 k}+p^{2 k-2}+\cdots+p^{2},
\end{aligned}
$$


for $k \geq 0$, so $\rho(-1)=1$ and $\rho(0)=0$. For even arguments, $\rho(2 k)=r(2 k)$ as defined in [AR02, Def. 2.5].

In all of the following spectral sequences we know that $\lambda_{2}, t \mu_{2}$ and $\bar{\epsilon}_{1}$ are infinite cycles. For $\lambda_{2}$ and $\bar{\epsilon}_{1}$ this follows from the $C_{p^{n}}$-fixed point analogue of diagram (5.1), by [AR02, Prop. 2.8] and Lemma 5.4. For $t \mu_{2}$ it follows from [AR02, Prop. 4.8], by naturality.

Theorem 6.1. The $C_{p^{n}}$-Tate spectral sequence in $V(1)$-homotopy for $T H H(\ell / p)$ begins

$$
\hat{E}^{2}\left(C_{p^{n}}, \ell / p\right)=E\left(u_{n}, \lambda_{2}\right) \otimes \mathbb{F}_{p}\left\{1, \epsilon_{0}, \mu_{0}, \epsilon_{0} \mu_{0}, \ldots, \mu_{0}^{p-1}, \bar{\epsilon}_{1}\right\} \otimes P\left(t^{ \pm 1}, \mu_{2}\right)
$$

and converges to $V(1)_{*} T H H(\ell / p)^{t C_{p^{n}}}$. It is a module spectral sequence over the algebra spectral sequence $\hat{E}^{*}\left(C_{p^{n}}, \ell\right)$ converging to $V(1)_{*} T H H(\ell)^{t C_{p^{n}}}$.

There is an initial $d^{2}$-differential generated by

$$
d^{2}\left(\epsilon_{0} \mu_{0}^{i-1}\right)=t \mu_{0}^{i}
$$

for $0<i<p$. Next, there are $2 n$ families of even length differentials generated by

$$
d^{2 \rho(2 k-1)}\left(t^{p^{2 k-1}-p^{2 k}+i} \cdot \bar{\epsilon}_{1}\right) \doteq\left(t \mu_{2}\right)^{\rho(2 k-3)} \cdot t^{i}
$$

for $v_{p}(i)=2 k-2$, for each $k=1, \ldots, n$, and

$$
d^{2 \rho(2 k)}\left(t^{p^{2 k-1}-p^{2 k}}\right) \doteq \lambda_{2} \cdot t^{p^{2 k-1}} \cdot\left(t \mu_{2}\right)^{\rho(2 k-2)}
$$

for each $k=1, \ldots, n$. Finally, there is a differential of odd length generated by

$$
d^{2 \rho(2 n)+1}\left(u_{n} \cdot t^{-p^{2 n}}\right) \doteq\left(t \mu_{2}\right)^{\rho(2 n-2)+1} .
$$

We shall prove Theorem 6.1 by induction on $n$. The base case $n=1$ was covered by Proposition 5.7. We can therefore assume that Theorem 6.1 holds for some fixed $n \geq 1$, and must prove the corresponding statement for $n+1$. First we make the following deduction.

Corollary 6.2. The initial differential in the $C_{p^{n}}$-Tate spectral sequence in $V(1)$-homotopy for $\operatorname{TH} H(\ell / p)$ leaves

$$
\hat{E}^{3}\left(C_{p^{n}}, \ell / p\right)=E\left(u_{n}, \bar{\epsilon}_{1}, \lambda_{2}\right) \otimes P\left(t^{ \pm 1}, t \mu_{2}\right) .
$$

The next $2 n$ families of differentials leave the intermediate terms

$$
\begin{aligned}
\hat{E}^{2 \rho(1)+1}\left(C_{p^{n}}, \ell / p\right)= & E\left(u_{n}, \lambda_{2}\right) \otimes \mathbb{F}_{p}\left\{t^{-i} \mid 0<i<p\right\} \otimes P\left(t^{ \pm p}\right) \\
& \oplus E\left(u_{n}, \bar{\epsilon}_{1}, \lambda_{2}\right) \otimes P\left(t^{ \pm p}, t \mu_{2}\right)
\end{aligned}
$$

$($ for $m=1)$, 


$$
\begin{aligned}
\hat{E}^{2 \rho(2 m-1)+1}\left(C_{p^{n}}, \ell / p\right)=E\left(u_{n}, \lambda_{2}\right) \otimes \mathbb{F}_{p}\left\{t^{-i} \mid 0<i<p\right\} \otimes P\left(t^{ \pm p^{2}}\right) \\
\oplus \bigoplus_{k=2}^{m} E\left(u_{n}, \lambda_{2}\right) \otimes \mathbb{F}_{p}\left\{t^{j} \mid j \in \mathbb{Z}, v_{p}(j)=2 k-2\right\} \otimes P_{\rho(2 k-3)}\left(t \mu_{2}\right) \\
\oplus \bigoplus_{k=2}^{m-1} E\left(u_{n}, \bar{\epsilon}_{1}\right) \otimes \mathbb{F}_{p}\left\{t^{j} \lambda_{2} \mid j \in \mathbb{Z}, v_{p}(j)=2 k-1\right\} \otimes P_{\rho(2 k-2)}\left(t \mu_{2}\right) \\
\oplus E\left(u_{n}, \bar{\epsilon}_{1}, \lambda_{2}\right) \otimes P\left(t^{ \pm p^{2 m-1}}, t \mu_{2}\right)
\end{aligned}
$$

for $m=2, \ldots, n$, and

$$
\begin{aligned}
\hat{E}^{2 \rho(2 m)+1}\left(C_{p^{n}}, \ell / p\right)=E\left(u_{n}, \lambda_{2}\right) \otimes \mathbb{F}_{p}\left\{t^{-i} \mid 0<i<p\right\} \otimes P\left(t^{ \pm p^{2}}\right) \\
\oplus \bigoplus_{k=2}^{m} E\left(u_{n}, \lambda_{2}\right) \otimes \mathbb{F}_{p}\left\{t^{j} \mid j \in \mathbb{Z}, v_{p}(j)=2 k-2\right\} \otimes P_{\rho(2 k-3)}\left(t \mu_{2}\right) \\
\oplus \bigoplus_{k=2}^{m} E\left(u_{n}, \bar{\epsilon}_{1}\right) \otimes \mathbb{F}_{p}\left\{t^{j} \lambda_{2} \mid j \in \mathbb{Z}, v_{p}(j)=2 k-1\right\} \otimes P_{\rho(2 k-2)}\left(t \mu_{2}\right) \\
\oplus E\left(u_{n}, \bar{\epsilon}_{1}, \lambda_{2}\right) \otimes P\left(t^{ \pm p^{2 m}}, t \mu_{2}\right)
\end{aligned}
$$

for $m=1, \ldots, n$. The final differential leaves the $E^{2 \rho(2 n)+2}=E^{\infty}$-term, equal to

$$
\begin{aligned}
\hat{E}^{\infty}\left(C_{p^{n}}, \ell / p\right)= & E\left(u_{n}, \lambda_{2}\right) \otimes \mathbb{F}_{p}\left\{t^{-i} \mid 0<i<p\right\} \otimes P\left(t^{ \pm p^{2}}\right) \\
& \oplus \bigoplus_{k=2}^{n} E\left(u_{n}, \lambda_{2}\right) \otimes \mathbb{F}_{p}\left\{t^{j} \mid j \in \mathbb{Z}, v_{p}(j)=2 k-2\right\} \otimes P_{\rho(2 k-3)}\left(t \mu_{2}\right) \\
& \oplus \bigoplus_{k=2}^{n} E\left(u_{n}, \bar{\epsilon}_{1}\right) \otimes \mathbb{F}_{p}\left\{t^{j} \lambda_{2} \mid j \in \mathbb{Z}, v_{p}(j)=2 k-1\right\} \otimes P_{\rho(2 k-2)}\left(t \mu_{2}\right) \\
& \oplus E\left(\bar{\epsilon}_{1}, \lambda_{2}\right) \otimes P\left(t^{ \pm p^{2 n}}\right) \otimes P_{\rho(2 n-2)+1}\left(t \mu_{2}\right) .
\end{aligned}
$$

Proof. The statements about the $E^{3}-, E^{2 \rho(1)+1}$ - and $E^{2 \rho(2)+1}$-terms are clear from Proposition 5.7. For each $m=2, \ldots, n$ we proceed by a secondary induction. The differential

$$
d^{2 \rho(2 m-1)}\left(t^{p^{2 m-1}-p^{2 m}+i} \cdot \bar{\epsilon}_{1}\right) \doteq\left(t \mu_{2}\right)^{\rho(2 m-3)} \cdot t^{i}
$$

for $v_{p}(i)=2 m-2$ is nontrivial only on the summand

$$
E\left(u_{n}, \bar{\epsilon}_{1}, \lambda_{2}\right) \otimes P\left(t^{ \pm p^{2 m-2}}, t \mu_{2}\right)
$$

of the $E^{2 \rho(2 m-2)+1}=E^{2 \rho(2 m-1)}$-term, with homology

$$
\begin{aligned}
E\left(u_{n}, \lambda_{2}\right) \otimes \mathbb{F}_{p}\left\{t^{j} \mid j \in \mathbb{Z}, v_{p}(j)=2 m-2\right\} & \otimes P_{\rho(2 m-3)}\left(t \mu_{2}\right) \\
& \oplus E\left(u_{n}, \bar{\epsilon}_{1}, \lambda_{2}\right) \otimes P\left(t^{ \pm p^{2 m-1}}, t \mu_{2}\right) .
\end{aligned}
$$


This gives the stated $E^{2 \rho(2 m-1)+1}$-term. Similarly, the differential

$$
d^{2 \rho(2 m)}\left(t^{p^{2 m-1}-p^{2 m}}\right) \doteq \lambda_{2} \cdot t^{p^{2 m-1}} \cdot\left(t \mu_{2}\right)^{\rho(2 m-2)}
$$

is nontrivial only on the summand

$$
E\left(u_{n}, \bar{\epsilon}_{1}, \lambda_{2}\right) \otimes P\left(t^{ \pm p^{2 m-1}}, t \mu_{2}\right)
$$

of the $E^{2 \rho(2 m-1)+1}=E^{2 \rho(2 m)}$-term, with homology

$$
\begin{aligned}
& E\left(u_{n}, \bar{\epsilon}_{1}\right) \otimes \mathbb{F}_{p}\left\{t^{j} \lambda_{2} \mid j \in \mathbb{Z}, v_{p}(j)=2 m-1\right\} \otimes P_{\rho(2 m-2)}\left(t \mu_{2}\right) \\
& \oplus E\left(u_{n}, \bar{\epsilon}_{1}, \lambda_{2}\right) \otimes P\left(t^{ \pm p^{2 m}}, t \mu_{2}\right) .
\end{aligned}
$$

This gives the stated $E^{2 \rho(2 m)+1}$-term. The final differential

$$
d^{2 \rho(2 n)+1}\left(u_{n} \cdot t^{-p^{2 n}}\right) \doteq\left(t \mu_{2}\right)^{\rho(2 n-2)+1}
$$

is nontrivial only on the summand

$$
E\left(u_{n}, \bar{\epsilon}_{1}, \lambda_{2}\right) \otimes P\left(t^{ \pm p^{2 n}}, t \mu_{2}\right)
$$

of the $E^{2 \rho(2 n)+1}$-term, with homology

$$
E\left(\bar{\epsilon}_{1}, \lambda_{2}\right) \otimes P\left(t^{ \pm p^{2 n}}\right) \otimes P_{\rho(2 n-2)+1}\left(t \mu_{2}\right) .
$$

This gives the stated $E^{2 \rho(2 n)+2}$-term. At this stage there is no room for any further differentials, since the spectral sequence is concentrated in a narrower horizontal band than the vertical height of the following differentials.

Next we compare the $C_{p^{n}}$-Tate spectral sequence with the $C_{p^{n}}$-homotopy fixed point spectral sequence obtained by restricting the $E^{2}$-term to the second quadrant $(s \leq 0$, $t \geq 0$ ). It is algebraically easier to handle the latter after inverting $\mu_{2}$, which can be interpreted as comparing $T H H(\ell / p)$ with its $C_{p}$-Tate construction.

In general, there is a commutative diagram

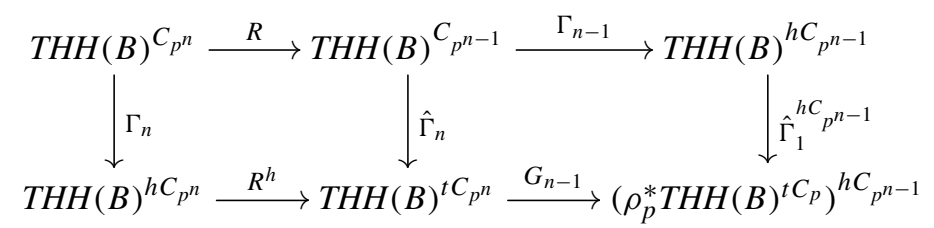

Here $\rho_{p}^{*} T H H(B)^{t C_{p}}$ is a notation for the $S^{1}$-spectrum obtained from the $S^{1} / C_{p}$-spectrum $T H H(B)^{t C_{p}}$ via the $p$-th root isomorphism $\rho_{p}: S^{1} \rightarrow S^{1} / C_{p}$, and $G_{n-1}$ is the comparison map from the $C_{p^{n-1}}$-fixed points to the $C_{p^{n-1}}$-homotopy fixed points of $\rho_{p}^{*} T H H(B)^{t} C_{p}$, in view of the identification

$$
\left(\rho_{p}^{*} T H H(B)^{t C_{p}}\right)^{C_{p^{n-1}}}=T H H(B)^{t C_{p^{n}}} .
$$


We are of course considering the case $B=\ell / p$. In $V(1)$-homotopy all four maps with labels containing $\Gamma$ are $(2 p-2)$-coconnected, by Corollary 5.9 , so $G_{n-1}$ is at least $(2 p-1)$-coconnected. (We shall see in Lemma 6.8 that $V(1)_{*} G_{n-1}$ is an isomorphism in all degrees.) By Proposition 5.8 the map $\hat{\Gamma}_{1}$ precisely inverts $\mu_{2}$, so the $E^{2}$-term of the $C_{p^{n}}$-homotopy fixed point spectral sequence in $V(1)$-homotopy for $T H H(\ell / p)^{t C_{p}}$ is obtained by inverting $\mu_{2}$ in $E^{2}\left(C_{p^{n}}, \ell / p\right)$. We denote this spectral sequence by $\mu_{2}^{-1} E^{*}\left(C_{p^{n}}, \ell / p\right)$, even though in later terms only a power of $\mu_{2}$ is present.

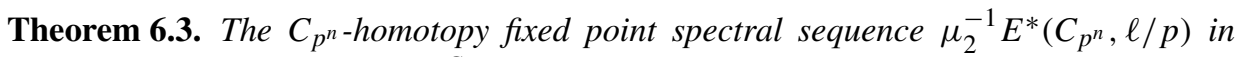
$V(1)$-homotopy for $\operatorname{THH}(\ell / p)^{t C_{p}}$ begins

$$
\mu_{2}^{-1} E^{2}\left(C_{p^{n}}, \ell / p\right)=E\left(u_{n}, \lambda_{2}\right) \otimes \mathbb{F}_{p}\left\{1, \epsilon_{0}, \mu_{0}, \epsilon_{0} \mu_{0}, \ldots, \mu_{0}^{p-1}, \bar{\epsilon}_{1}\right\} \otimes P\left(t, \mu_{2}^{ \pm 1}\right)
$$

and converges to $V(1)_{*}\left(\rho_{p}^{*} T H H(\ell / p)^{t C_{p}}\right)^{h C_{p^{n}}}$, which receives a $(2 p-2)$-coconnected map $\left(\hat{\Gamma}_{1}\right)^{h C_{p^{n}}}$ from $V(1)_{*} T H H(\ell / p)^{h C_{p^{n}}}$. There is an initial $d^{2}$-differential generated by

$$
d^{2}\left(\epsilon_{0} \mu_{0}^{i-1}\right)=t \mu_{0}^{i}
$$

for $0<i<p$. Next, there are $2 n$ families of even length differentials generated by

$$
d^{2 \rho(2 k-1)}\left(\mu_{2}^{p^{2 k}-p^{2 k-1}+j} \cdot \bar{\epsilon}_{1}\right) \doteq\left(t \mu_{2}\right)^{\rho(2 k-1)} \cdot \mu_{2}^{j}
$$

for $v_{p}(j)=2 k-2$, for each $k=1, \ldots, n$, and

$$
d^{2 \rho(2 k)}\left(\mu_{2}^{p^{2 k}-p^{2 k-1}}\right) \doteq \lambda_{2} \cdot \mu_{2}^{-p^{2 k-1}} \cdot\left(t \mu_{2}\right)^{\rho(2 k)}
$$

for each $k=1, \ldots, n$. Finally, there is a differential of odd length generated by

$$
d^{2 \rho(2 n)+1}\left(u_{n} \cdot \mu_{2}^{p^{2 n}}\right) \doteq\left(t \mu_{2}\right)^{\rho(2 n)+1} .
$$

Proof. The differential pattern follows from Theorem 6.1 by naturality with respect to the maps of spectral sequences

$$
\mu_{2}^{-1} E^{*}\left(C_{p^{n}}, \ell / p\right) \stackrel{\hat{\Gamma}_{1}^{h C_{p^{n}}}}{\longleftarrow} E^{*}\left(C_{p^{n}}, \ell / p\right) \stackrel{R^{h}}{\longrightarrow} \hat{E}^{*}\left(C_{p^{n}}, \ell / p\right)
$$

induced by $\hat{\Gamma}_{1}^{h C_{p^{n}}}$ and $R^{h}$. The first inverts $\mu_{2}$ and the second inverts $t$, at the level of $E^{2}$-terms. We are also using that $t \mu_{2}$, the image of $v_{2}$, multiplies as an infinite cycle in all of these spectral sequences.

Corollary 6.4. The initial differential in the $C_{p^{n}}$-homotopy fixed point spectral sequence in $V(1)$-homotopy for $\operatorname{THH}(\ell / p)^{t C_{p}}$ leaves

$$
\begin{aligned}
\mu_{2}^{-1} E^{3}\left(C_{p^{n}}, \ell / p\right)= & E\left(u_{n}, \lambda_{2}\right) \otimes \mathbb{F}_{p}\left\{\mu_{0}^{i} \mid 0<i<p\right\} \otimes P\left(\mu_{2}^{ \pm 1}\right) \\
& \oplus E\left(u_{n}, \bar{\epsilon}_{1}, \lambda_{2}\right) \otimes P\left(\mu_{2}^{ \pm 1}, t \mu_{2}\right) .
\end{aligned}
$$


The next $2 n$ families of differentials leave the intermediate terms

$$
\begin{aligned}
\mu_{2}^{-1} E^{2 \rho(2 m-1)+1}\left(C_{p^{n}}, \ell / p\right)=E\left(u_{n}, \lambda_{2}\right) \otimes \mathbb{F}_{p}\left\{\mu_{0}^{i} \mid 0<i<p\right\} \otimes P\left(\mu_{2}^{ \pm 1}\right) \\
\oplus \bigoplus_{k=1}^{m} E\left(u_{n}, \lambda_{2}\right) \otimes \mathbb{F}_{p}\left\{\mu_{2}^{j} \mid j \in \mathbb{Z}, v_{p}(j)=2 k-2\right\} \otimes P_{\rho(2 k-1)}\left(t \mu_{2}\right) \\
\oplus \bigoplus_{k=1}^{m-1} E\left(u_{n}, \bar{\epsilon}_{1}\right) \otimes \mathbb{F}_{p}\left\{\lambda_{2} \mu_{2}^{j} \mid j \in \mathbb{Z}, v_{p}(j)=2 k-1\right\} \otimes P_{\rho(2 k)}\left(t \mu_{2}\right) \\
\oplus E\left(u_{n}, \bar{\epsilon}_{1}, \lambda_{2}\right) \otimes P\left(\mu_{2}^{ \pm p^{2 m-1}}, t \mu_{2}\right)
\end{aligned}
$$

and

$$
\begin{aligned}
\mu_{2}^{-1} E^{2 \rho(2 m)+1}\left(C_{p^{n}}, \ell / p\right)=E\left(u_{n}, \lambda_{2}\right) \otimes \mathbb{F}_{p}\left\{\mu_{0}^{i} \mid 0<i<p\right\} \otimes P\left(\mu_{2}^{ \pm 1}\right) \\
\oplus \bigoplus_{k=1}^{m} E\left(u_{n}, \lambda_{2}\right) \otimes \mathbb{F}_{p}\left\{\mu_{2}^{j} \mid j \in \mathbb{Z}, v_{p}(j)=2 k-2\right\} \otimes P_{\rho(2 k-1)}\left(t \mu_{2}\right) \\
\oplus \bigoplus_{k=1}^{m} E\left(u_{n}, \bar{\epsilon}_{1}\right) \otimes \mathbb{F}_{p}\left\{\lambda_{2} \mu_{2}^{j} \mid j \in \mathbb{Z}, v_{p}(j)=2 k-1\right\} \otimes P_{\rho(2 k)}\left(t \mu_{2}\right) \\
\oplus E\left(u_{n}, \bar{\epsilon}_{1}, \lambda_{2}\right) \otimes P\left(\mu_{2}^{ \pm p^{2 m}}, t \mu_{2}\right)
\end{aligned}
$$

for $m=1, \ldots, n$. The final differential leaves the $E^{2 \rho(2 n)+2}=E^{\infty}$-term, equal to

$$
\begin{aligned}
\mu_{2}^{-1} E^{\infty}\left(C_{p^{n}}, \ell / p\right) & =E\left(u_{n}, \lambda_{2}\right) \otimes \mathbb{F}_{p}\left\{\mu_{0}^{i} \mid 0<i<p\right\} \otimes P\left(\mu_{2}^{ \pm 1}\right) \\
& \oplus \bigoplus_{k=1}^{n} E\left(u_{n}, \lambda_{2}\right) \otimes \mathbb{F}_{p}\left\{\mu_{2}^{j} \mid j \in \mathbb{Z}, v_{p}(j)=2 k-2\right\} \otimes P_{\rho(2 k-1)}\left(t \mu_{2}\right) \\
& \oplus \bigoplus_{k=1}^{n} E\left(u_{n}, \bar{\epsilon}_{1}\right) \otimes \mathbb{F}_{p}\left\{\lambda_{2} \mu_{2}^{j} \mid j \in \mathbb{Z}, v_{p}(j)=2 k-1\right\} \otimes P_{\rho(2 k)}\left(t \mu_{2}\right) \\
& \oplus E\left(\bar{\epsilon}_{1}, \lambda_{2}\right) \otimes P\left(\mu_{2}^{ \pm p^{2 n}}\right) \otimes P_{\rho(2 n)+1}\left(t \mu_{2}\right) .
\end{aligned}
$$

Proof. The computation of the $E^{3}$-term from the $E^{2}$-term is straightforward. The rest of the proof goes by a secondary induction on $m=1, \ldots, n$, very much like the proof of Corollary 6.2. The differential

$$
d^{2 \rho(2 m-1)}\left(\mu_{2}^{p^{2 m}-p^{2 m-1}+j} \cdot \bar{\epsilon}_{1}\right) \doteq\left(t \mu_{2}\right)^{\rho(2 m-1)} \cdot \mu_{2}^{j}
$$

for $v_{p}(j)=2 m-2$ is nontrivial only on the summand

$$
E\left(u_{n}, \bar{\epsilon}_{1}, \lambda_{2}\right) \otimes P\left(\mu_{2}^{ \pm p^{2 m-2}}, t \mu_{2}\right)
$$

of the $E^{3}=E^{2 \rho(1)}$-term (for $m=1$ ), resp. the $E^{2 \rho(2 m-2)+1}=E^{2 \rho(2 m-1)}$-term (for $m=2, \ldots, n)$. Its homology is 


$$
\begin{aligned}
E\left(u_{n}, \lambda_{2}\right) \otimes \mathbb{F}_{p}\left\{\mu_{2}^{j} \mid j \in \mathbb{Z}, v_{p}(j)=2 m-\right. & 2\} \otimes P_{\rho(2 m-1)}\left(t \mu_{2}\right) \\
& \oplus E\left(u_{n}, \bar{\epsilon}_{1}, \lambda_{2}\right) \otimes P\left(\mu_{2}^{ \pm p^{2 m-1}}, t \mu_{2}\right),
\end{aligned}
$$

which gives the stated $E^{2 \rho(2 m-1)+1}$-term. The differential

$$
d^{2 \rho(2 m)}\left(\mu_{2}^{p^{2 m}-p^{2 m-1}}\right) \doteq \lambda_{2} \cdot \mu_{2}^{-p^{2 m-1}} \cdot\left(t \mu_{2}\right)^{\rho(2 m)}
$$

is nontrivial only on the summand

$$
E\left(u_{n}, \bar{\epsilon}_{1}, \lambda_{2}\right) \otimes P\left(\mu_{2}^{ \pm p^{2 m-1}}, t \mu_{2}\right)
$$

of the $E^{2 \rho(2 m-1)+1}=E^{2 \rho(2 m)}$-term, leaving

$$
\begin{aligned}
& E\left(u_{n}, \bar{\epsilon}_{1}\right) \otimes \mathbb{F}_{p}\left\{\lambda_{2} \mu_{2}^{j} \mid j \in \mathbb{Z}, v_{p}(j)=2 m-1\right\} \otimes P_{\rho(2 m)}\left(t \mu_{2}\right) \\
& \oplus E\left(u_{n}, \bar{\epsilon}_{1}, \lambda_{2}\right) \otimes P\left(\mu_{2}^{ \pm p^{2 m}}, t \mu_{2}\right) .
\end{aligned}
$$

This gives the stated $E^{2 \rho(2 m)+1}$-term. The final differential

$$
d^{2 \rho(2 n)+1}\left(u_{n} \cdot \mu_{2}^{p^{2 n}}\right) \doteq\left(t \mu_{2}\right)^{\rho(2 n)+1}
$$

is nontrivial only on the summand

$$
E\left(u_{n}, \bar{\epsilon}_{1}, \lambda_{2}\right) \otimes P\left(\mu_{2}^{ \pm p^{2 n}}, t \mu_{2}\right)
$$

of the $E^{2 \rho(2 n)+1}$-term, with homology

$$
E\left(\bar{\epsilon}_{1}, \lambda_{2}\right) \otimes P\left(\mu_{2}^{ \pm p^{2 n}}\right) \otimes P_{\rho(2 n)+1}\left(t \mu_{2}\right) .
$$

This gives the stated $E^{2 \rho(2 n)+2}$-term. There is no room for any further differentials, since the spectral sequence is concentrated in a narrower vertical band than the horizontal width of the following differentials, so $E^{2 \rho(2 n)+2}=E^{\infty}$.

Proof of Theorem 6.1. To make the inductive step to $C_{p^{n+1}}$, we use that the first $d^{r}$ differential of odd length in $\hat{E}^{*}\left(C_{p^{n}}, \ell / p\right)$ occurs for $r=r_{0}=2 \rho(2 n)+1$. It follows from [AR02, Lem. 5.2] that the terms $\hat{E}^{r}\left(C_{p^{n}}, \ell / p\right)$ and $\hat{E}^{r}\left(C_{p^{n+1}}, \ell / p\right)$ are isomorphic for $r \leq 2 \rho(2 n)+1$, via the Frobenius map (taking $t^{i}$ to $t^{i}$ ) in even columns and the Verschiebung map (taking $u_{n} t^{i}$ to $u_{n+1} t^{i}$ ) in odd columns. Furthermore, the differential $d^{2 \rho(2 n)+1}$ is zero in the latter spectral sequence. This proves the part of Theorem 6.1 for $n+1$ that concerns the differentials leading up to the term

$$
\begin{aligned}
& \hat{E}^{2 \rho(2 n)+2}\left(C_{p^{n+1}}, \ell / p\right)=E\left(u_{n+1}, \lambda_{2}\right) \otimes \mathbb{F}_{p}\left\{t^{-i} \mid 0<i<p\right\} \otimes P\left(t^{ \pm p^{2}}\right) \\
& \oplus \bigoplus_{k=2}^{n} E\left(u_{n+1}, \lambda_{2}\right) \otimes \mathbb{F}_{p}\left\{t^{j} \mid j \in \mathbb{Z}, v_{p}(j)=2 k-2\right\} \otimes P_{\rho(2 k-3)}\left(t \mu_{2}\right) \\
& \oplus \bigoplus_{k=2}^{n} E\left(u_{n+1}, \bar{\epsilon}_{1}\right) \otimes \mathbb{F}_{p}\left\{t^{j} \lambda_{2} \mid j \in \mathbb{Z}, v_{p}(j)=2 k-1\right\} \otimes P_{\rho(2 k-2)}\left(t \mu_{2}\right) \\
& \oplus E\left(u_{n+1}, \bar{\epsilon}_{1}, \lambda_{2}\right) \otimes P\left(t^{ \pm p^{2 n}}, t \mu_{2}\right) .
\end{aligned}
$$


Next we use the following commutative diagram, where we abbreviate $\operatorname{THH}(B)$ to $T(B)$ for typographical reasons:

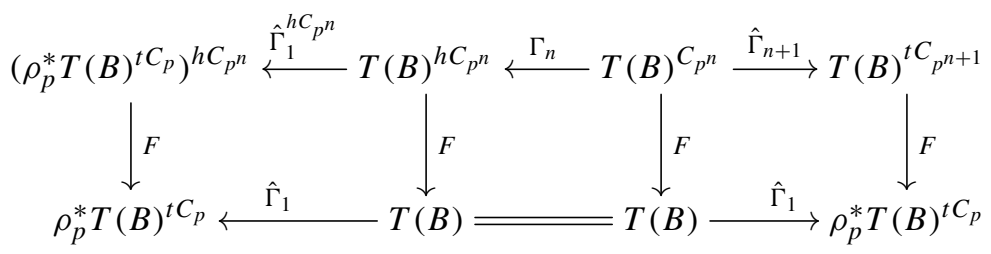

The horizontal maps all induce $(2 p-2)$-coconnected maps in $V(1)$-homotopy for $B=\ell / p$. Each $F$ is a Frobenius map, forgetting invariance under a $C_{p^{n}}$-action. Thus the map $\hat{\Gamma}_{n+1}$ to the right induces an isomorphism of $E\left(\lambda_{2}\right) \otimes P\left(v_{2}\right)$-modules in all degrees $*>2 p-2$ from $V(1)_{*} T H H(\ell / p) C_{p^{n}}$, implicitly identified to the left with the abutment of $\mu_{2}^{-1} E^{*}\left(C_{p^{n}}, \ell / p\right)$, to $V(1)_{*} T H H(\ell / p)^{t C_{p^{n+1}}}$, which is the abutment of $\hat{E}^{*}\left(C_{p^{n+1}}, \ell / p\right)$.

The diagram above ensures that the isomorphism induced by $\hat{\Gamma}_{n+1}$ is compatible with the one induced by $\hat{\Gamma}_{1}$. By Proposition 5.8 it takes $\bar{\epsilon}_{1}, \lambda_{2}$ and $\mu_{2}$ to $\bar{\epsilon}_{1}, \lambda_{2}$ and $t^{-p^{2}}$ up to a unit factor in $\mathbb{F}_{p}$, respectively, and similarly for monomials in these classes.

We focus on the summand

$$
E\left(u_{n}, \lambda_{2}\right) \otimes \mathbb{F}_{p}\left\{\mu_{2}^{j} \mid j \in \mathbb{Z}, v_{p}(j)=2 n-2\right\} \otimes P_{\rho(2 n-1)}\left(t \mu_{2}\right)
$$

in $\mu_{2}^{-1} E^{\infty}\left(C_{p^{n}}, \ell / p\right)$, abutting to $V(1)_{*} T H H(\ell / p){ }^{C_{p^{n}}}$ in degrees $>2 p-2$. In the $P\left(v_{2}\right)$ module structure on the abutment, each class $\mu_{2}^{j}$ with $v_{p}(j)=2 n-2, j>0$, generates a copy of $P_{\rho(2 n-1)}\left(v_{2}\right)$, since there are no permanent cycles in the same total degree as $y=\left(t \mu_{2}\right)^{\rho(2 n-1)} \cdot \mu_{2}^{j}$ that have lower (= more negative) homotopy fixed point filtration. See Lemma 6.5 below for the elementary verification. The $P\left(v_{2}\right)$-module isomorphism induced by $\hat{\Gamma}_{n+1}$ must take this to a copy of $P_{\rho(2 n-1)}\left(v_{2}\right)$ in $V(1)_{*} T H H(\ell / p)^{t C_{p^{n+1}}}$, generated by $t^{-p^{2} j}$.

Writing $i=-p^{2} j$, we deduce that for $v_{p}(i)=2 n, i<0$, the infinite cycle $z=$ $\left(t \mu_{2}\right)^{\rho(2 n-1)} \cdot t^{i}$ must represent zero in the abutment, and must therefore be hit by a differential $z=d^{r}(x)$ in the $C_{p^{n+1}}$-Tate spectral sequence. Here $r \geq 2 \rho(2 n)+2$.

Since $z$ generates a free copy of $P\left(t \mu_{2}\right)$ in the $E^{2 \rho(2 n)+2}$-term displayed in (6.2), and $d^{r}$ is $P\left(t \mu_{2}\right)$-linear, the class $x$ cannot be annihilated by any power of $t \mu_{2}$. This means that $x$ must be contained in the summand

$$
E\left(u_{n+1}, \bar{\epsilon}_{1}, \lambda_{2}\right) \otimes P\left(t^{ \pm p^{2 n}}, t \mu_{2}\right)
$$

of $\hat{E}^{2 \rho(2 n)+2}\left(C_{p^{n+1}}, \ell / p\right)$. By an elementary check of bidegrees (see Lemma 6.6 below), the only possibility is that $x$ has vertical degree $2 p-1$, so that we have differentials

$$
d^{2 \rho(2 n+1)}\left(t^{p^{2 n+1}-p^{2 n+2}+i} \cdot \bar{\epsilon}_{1}\right) \doteq\left(t \mu_{2}\right)^{\rho(2 n-1)} \cdot t^{i}
$$


for all $i<0$ with $v_{p}(i)=2 n$. The cases $i>0$ follow by the module structure over the $C_{p^{n+1}}$-Tate spectral sequence for $\ell$. The remaining two differentials,

$$
d^{2 \rho(2 n+2)}\left(t^{p^{2 n+1}-p^{2 n+2}}\right) \doteq \lambda_{2} \cdot t^{p^{2 n+1}} \cdot\left(t \mu_{2}\right)^{\rho(2 n)}
$$

and

$$
d^{2 \rho(2 n+2)+1}\left(u_{n+1} \cdot t^{-p^{2 n+2}}\right) \doteq\left(t \mu_{2}\right)^{\rho(2 n)+1},
$$

are also present in the $C_{p^{n+1}}$-Tate spectral sequence for $\ell$ (see [AR02, Th 6.1]), hence follow in the present case by the module structure. With this we have established the complete differential pattern asserted by Theorem 6.1.

Lemma 6.5. For $j \in \mathbb{Z}$ with $v_{p}(j)=2 n-2$, where $n \geq 1$, there are no classes in $\mu_{2}^{-1} E^{\infty}\left(C_{p^{n}}, \ell / p\right)$ in the same total degree as $y=\left(t \mu_{2}\right)^{\rho(2 n-1)} \cdot \mu_{2}^{j}$ that have lower homotopy fixed point filtration.

Proof. The total degree of $y$ is $2\left(p^{2 n+2}-p^{2 n+1}+p-1\right)+2 p^{2} j \equiv 2 p-2 \bmod 2 p^{2 n}$, which is even.

Looking at the formula for $\mu_{2}^{-1} E^{\infty}\left(C_{p^{n}}, \ell / p\right)$ in Corollary 6.4, the classes of lower filtration than $y$ all lie in the terms

$$
E\left(u_{n}, \bar{\epsilon}_{1}\right) \otimes \mathbb{F}_{p}\left\{\lambda_{2} \mu_{2}^{i} \mid j \in \mathbb{Z}, v_{p}(i)=2 n-1\right\} \otimes P_{\rho(2 n)}\left(t \mu_{2}\right)
$$

and

$$
E\left(\bar{\epsilon}_{1}, \lambda_{2}\right) \otimes P\left(\mu_{2}^{ \pm p^{2 n}}\right) \otimes P_{\rho(2 n)+1}\left(t \mu_{2}\right) .
$$

Those in even total degree and of lower filtration than $y$ are

$$
u_{n} \lambda_{2} \cdot \mu_{2}^{i}\left(t \mu_{2}\right)^{e}, \quad \bar{\epsilon}_{1} \lambda_{2} \cdot \mu_{2}^{i}\left(t \mu_{2}\right)^{e}
$$

with $v_{p}(i)=2 n-1, \rho(2 n-1)<e<\rho(2 n)$, and

$$
\mu_{2}^{i}\left(t \mu_{2}\right)^{e}, \quad \bar{\epsilon}_{1} \lambda_{2} \cdot \mu_{2}^{i}\left(t \mu_{2}\right)^{e}
$$

with $v_{p}(i) \geq 2 n, \rho(2 n-1)<e \leq \rho(2 n)$.

The total degree of $u_{n} \lambda_{2} \cdot \mu_{2}^{i}\left(t \mu_{2}\right)^{e}$ for $v_{p}(i)=2 n-1$ is $(-1)+\left(2 p^{2}-1\right)+2 p^{2} i+$ $\left(2 p^{2}-2\right) e \equiv\left(2 p^{2}-2\right)(e+1) \bmod 2 p^{2 n}$. For this to agree with the total degree of $y$, we must have $2 p-2 \equiv\left(2 p^{2}-2\right)(e+1) \bmod 2 p^{2 n}$, so $e+1 \equiv 1 /(1+p) \bmod p^{2 n}$ and $e \equiv \rho(2 n-1)-1 \bmod p^{2 n}$. There is no such $e$ with $\rho(2 n-1)<e<\rho(2 n)$.

The total degree of $\bar{\epsilon}_{1} \lambda_{2} \cdot \mu_{2}^{i}\left(t \mu_{2}\right)^{e}$ for $v_{p}(i)=2 n-1$ is $(2 p-1)+\left(2 p^{2}-1\right)+$ $2 p^{2} i+\left(2 p^{2}-2\right) e \equiv 2 p+\left(2 p^{2}-2\right)(e+1) \bmod 2 p^{2 n}$. To agree with that of $y$, we must have $2 p-2 \equiv 2 p+\left(2 p^{2}-2\right)(e+1) \bmod 2 p^{2 n}$, so $(e+1) \equiv 1 /\left(1-p^{2}\right) \bmod p^{2 n}$ and $e \equiv \rho(2 n) \bmod p^{2 n}$. There is no such $e$ with $\rho(2 n-1)<e<\rho(2 n)$.

The total degree of $\mu_{2}^{i}\left(t \mu_{2}\right)^{e}$ for $v_{p}(i) \geq 2 n$ is $2 p^{2} i+\left(2 p^{2}-2\right) e \equiv\left(2 p^{2}-2\right) e$ $\bmod 2 p^{2 n}$. To agree with that of $y$, we must have $(2 p-2) \equiv\left(2 p^{2}-2\right) e \bmod 2 p^{2 n}$, so $e \equiv 1 /(1+p) \equiv \rho(2 n-1) \bmod p^{2 n}$. There is no such $e$ with $\rho(2 n-1)<e \leq \rho(2 n)$.

The total degree of $\bar{\epsilon}_{1} \lambda_{2} \cdot \mu_{2}^{i}\left(t \mu_{2}\right)^{e}$ for $v_{p}(i) \geq 2 n$ is $(2 p-1)+\left(2 p^{2}-1\right)+2 p^{2} i+$ $\left(2 p^{2}-2\right) e$. To agree modulo $2 p^{2 n}$ with that of $y$, we must have $e \equiv \rho(2 n) \bmod p^{2 n}$. The 
only such $e$ with $\rho(2 n-1)<e \leq \rho(2 n)$ is $e=\rho(2 n)$. But in that case, the total degree of $\bar{\epsilon}_{1} \lambda_{2} \cdot \mu_{2}^{i}\left(t \mu_{2}\right)^{e}$ is $2 p+2 p^{2} i+\left(2 p^{2}-2\right)(\rho(2 n)+1)=2\left(p^{2 n+2}+p-1\right)+2 p^{2} i$. To be equal to that of $y$, we must have $2 p^{2} i+2 p^{2 n+1}=2 p^{2} j$, which is impossible for $v_{p}(i) \geq 2 n$ and $v_{p}(j)=2 n-2$.

Lemma 6.6. For $v_{p}(i)=2 n, n \geq 1$ and $z=\left(t \mu_{2}\right)^{\rho(2 n-1)} \cdot t^{i}$, the only class in

$$
E\left(u_{n+1}, \bar{\epsilon}_{1}, \lambda_{2}\right) \otimes P\left(t^{ \pm p^{2 n}}, t \mu_{2}\right)
$$

that can support a nonzero differential $d^{r}(x)=z$ for $r \geq 2 \rho(2 n)+2$ is (a unit times)

$$
x=t^{p^{2 n+1}-p^{2 n+2}+i} \cdot \bar{\epsilon}_{1} .
$$

Proof. The class $z$ has total degree $\left(2 p^{2}-2\right) \rho(2 n-1)-2 i=2 p^{2 n+2}-2 p^{2 n+1}+$ $2 p-2-2 i \equiv 2 p-2 \bmod 2 p^{2 n}$, which is even, and vertical degree $2 p^{2} \rho(2 n-1)$. Hence $x$ has odd total degree, and vertical degree at most $2 p^{2} \rho(2 n-1)-2 \rho(2 n)-1=$ $2 p^{2 n+2}-2 p^{2 n+1}-\cdots-2 p^{3}-1$. This leaves the possibilities

$$
u_{n+1} \cdot t^{j}\left(t \mu_{2}\right)^{e}, \quad \bar{\epsilon}_{1} \cdot t^{j}\left(t \mu_{2}\right)^{e}, \quad \lambda_{2} \cdot t^{j}\left(t \mu_{2}\right)^{e}
$$

with $v_{p}(j) \geq 2 n$ and $0 \leq e<p^{2 n}-p^{2 n-1}-\cdots-p=\rho(2 n-1)-\rho(2 n-2)-1$, and

$$
u_{n+1} \bar{\epsilon}_{1} \lambda_{2} \cdot t^{j}\left(t \mu_{2}\right)^{e}
$$

with $v_{p}(j) \geq 2 n$ and $0 \leq e<p^{2 n}-p^{2 n-1}-\cdots-p-1=\rho(2 n-1)-\rho(2 n-2)-2$.

The total degree of $x$ must be one more than the total degree of $z$, hence is congruent to $2 p-1$ modulo $2 p^{2 n}$.

The total degree of $u_{n+1} \cdot t^{j}\left(t \mu_{2}\right)^{e}$ is $-1-2 j+\left(2 p^{2}-2\right) e \equiv-1+\left(2 p^{2}-2\right) e$ $\bmod 2 p^{2 n}$. To have $2 p-1 \equiv-1+\left(2 p^{2}-2\right) e \bmod 2 p^{2 n}$ we must have $e \equiv-p /\left(1-p^{2}\right) \equiv$ $p^{2 n}-p^{2 n-1}-\cdots-p \bmod p^{2 n}$, which does not happen for $e$ in the allowable range.

The total degree of $\lambda_{2} \cdot t^{j}\left(t \mu_{2}\right)^{e}$ is $\left(2 p^{2}-1\right)-2 j+\left(2 p^{2}-2\right) e \equiv\left(2 p^{2}-1\right)+\left(2 p^{2}-2\right) e$ $\bmod 2 p^{2 n}$. To have $2 p-1 \equiv\left(2 p^{2}-1\right)+\left(2 p^{2}-2\right) e \bmod 2 p^{2 n}$ we must have $e \equiv$ $-p /(1+p) \equiv \rho(2 n-1)-1 \bmod p^{2 n}$, which does not happen.

The total degree of $u_{n+1} \bar{\epsilon}_{1} \lambda_{2} \cdot t^{j}\left(t \mu_{2}\right)^{e}$ is $-1+(2 p-1)+\left(2 p^{2}-1\right)-2 j+\left(2 p^{2}-2\right) e \equiv$ $(2 p-1)+\left(2 p^{2}-2\right)(e+1) \bmod 2 p^{2 n}$. To have $2 p-1 \equiv(2 p-1)+\left(2 p^{2}-2\right)(e+1)$ $\bmod 2 p^{2 n}$ we must have $e+1 \equiv 0 \bmod p^{2 n}$, so $e \equiv p^{2 n}-1 \bmod p^{2 n}$, which does not happen.

The total degree of $\bar{\epsilon}_{1} \cdot t^{j}\left(t \mu_{2}\right)^{e}$ is $(2 p-1)-2 j+\left(2 p^{2}-2\right) e \equiv(2 p-1)+\left(2 p^{2}-2\right) e$ $\bmod 2 p^{2 n}$. To have $2 p-1 \equiv(2 p-1)+\left(2 p^{2}-2\right) e \bmod 2 p^{2 n}$, we must have $e \equiv 0$ $\bmod p^{2 n}$, so $e=0$ is the only possibility in the allowable range. In that case, a check of total degrees shows that we must have $j=p^{2 n+1}-p^{2 n+2}+i$.

Corollary 6.7. $V(1)_{*} T H H(\ell / p)^{C_{p^{n}}}$ is finite in each degree.

Proof. This is clear by inspection of the $E^{\infty}$-term in Corollary 6.2. 
Lemma 6.8. The map $G_{n}$ induces an isomorphism

$$
V(1)_{*} T H H(\ell / p)^{t C_{p^{n+1}}} \stackrel{\cong}{\rightarrow} V(1)_{*}\left(\rho_{p}^{*} T H H(\ell / p)^{t C_{p}}\right)^{h C_{p^{n}}}
$$

in all degrees. In the limit over the Frobenius maps $F$, there is a map $G$ inducing an isomorphism

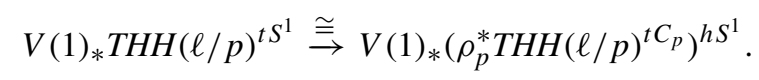

Proof. As remarked after diagram (6.1), $G_{n}$ induces an isomorphism in $V(1)$-homotopy above degree $2 p-2$. The permanent cycle $t^{-p^{2 n+2}}$ in $\hat{E}^{\infty}\left(C_{p^{n+1}}, \ell\right)$ acts invertibly on $\hat{E}^{\infty}\left(C_{p^{n+1}}, \ell / p\right)$, and its image $G_{n}\left(t^{-p^{2 n+2}}\right)=\mu_{2}^{p^{2 n}}$ in $\mu_{2}^{-1} E^{\infty}\left(C_{p^{n}}, \ell\right)$ acts invertibly on $\mu_{2}^{-1} E^{\infty}\left(C_{p^{n}}, \ell / p\right)$. Therefore the module action derived from the $\ell$-algebra structure on $\ell / p$ ensures that $G_{n}$ induces isomorphisms in $V(1)$-homotopy in all degrees.

Theorem 6.9. The isomorphism (6.4) admits the following description at the associated graded level:

(a) The associated graded of $V(1)_{*} T H H(\ell / p)^{t S^{1}}$ for the $S^{1}$-Tate spectral sequence is

$$
\begin{aligned}
\hat{E}^{\infty}\left(S^{1}, \ell / p\right)= & E\left(\lambda_{2}\right) \otimes \mathbb{F}_{p}\left\{t^{-i} \mid 0<i<p\right\} \otimes P\left(t^{ \pm p^{2}}\right) \\
& \oplus \bigoplus_{k \geq 2} E\left(\lambda_{2}\right) \otimes \mathbb{F}_{p}\left\{t^{j} \mid j \in \mathbb{Z}, v_{p}(j)=2 k-2\right\} \otimes P_{\rho(2 k-3)}\left(t \mu_{2}\right) \\
& \oplus \bigoplus_{k \geq 2} E\left(\bar{\epsilon}_{1}\right) \otimes \mathbb{F}_{p}\left\{t^{j} \lambda_{2} \mid j \in \mathbb{Z}, v_{p}(j)=2 k-1\right\} \otimes P_{\rho(2 k-2)}\left(t \mu_{2}\right) \\
& \oplus E\left(\bar{\epsilon}_{1}, \lambda_{2}\right) \otimes P\left(t \mu_{2}\right) .
\end{aligned}
$$

(b) The associated graded of $V(1)_{*} T H H(\ell / p)^{h S^{1}}$ for the $S^{1}$-homotopy fixed point spectral sequence maps by a $(2 p-2)$-coconnected map to

$$
\begin{aligned}
\mu_{2}^{-1} E^{\infty}\left(S^{1}, \ell / p\right)= & E\left(\lambda_{2}\right) \otimes \mathbb{F}_{p}\left\{\mu_{0}^{i} \mid 0<i<p\right\} \otimes P\left(\mu_{2}^{ \pm 1}\right) \\
& \oplus \bigoplus_{k \geq 1} E\left(\lambda_{2}\right) \otimes \mathbb{F}_{p}\left\{\mu_{2}^{j} \mid j \in \mathbb{Z}, v_{p}(j)=2 k-2\right\} \otimes P_{\rho(2 k-1)}\left(t \mu_{2}\right) \\
& \oplus \bigoplus_{k \geq 1} E\left(\bar{\epsilon}_{1}\right) \otimes \mathbb{F}_{p}\left\{\lambda_{2} \mu_{2}^{j} \mid j \in \mathbb{Z}, v_{p}(j)=2 k-1\right\} \otimes P_{\rho(2 k)}\left(t \mu_{2}\right) \\
& \oplus E\left(\bar{\epsilon}_{1}, \lambda_{2}\right) \otimes P\left(t \mu_{2}\right) .
\end{aligned}
$$

(c) The isomorphism from (a) to (b) induced by $G$ takes $t^{-i}$ to $\mu_{0}^{i}$ for $0<i<p$ and $t^{i}$ to $\mu_{2}^{j}$ for $i+p^{2} j=0$, up to a unit factor in $\mathbb{F}_{p}$. Furthermore, it takes multiples by $\bar{\epsilon}_{1}$, $\lambda_{2}$ or $t \mu_{2}$ in the source to the same multiples in the target, up to a unit factor in $\mathbb{F}_{p}$.

Proof. Claims (a) and (b) follow by passage to the limit over $n$ from Corollaries 6.2 and 6.4. Claim (c) follows by passage to the same limit from the formulas for the isomorphism induced by $\hat{\Gamma}_{n+1}$, which were given below diagram (6.3). 


\section{Topological cyclic homology}

By definition, there is a fibre sequence

$$
T C(B ; p) \stackrel{\pi}{\rightarrow} T F(B ; p) \stackrel{R-1}{\longrightarrow} T F(B ; p) \rightarrow \Sigma T C(B ; p)
$$

inducing a long exact sequence

$$
\cdots \stackrel{\partial}{\rightarrow} V(1)_{*} T C(B ; p) \stackrel{\pi}{\rightarrow} V(1)_{*} T F(B ; p) \stackrel{R_{*}-1}{\longrightarrow} V(1)_{*} T F(B ; p) \stackrel{\partial}{\rightarrow} \cdots
$$

in $V$ (1)-homotopy. By Corollary 5.9, there are $(2 p-2)$-coconnected maps $\Gamma$ and $\hat{\Gamma}$ from $V(1)_{*} T F(\ell / p ; p)$ to $V(1)_{*} T H H(\ell / p)^{h S^{1}}$ and $V(1)_{*} T H H(\ell / p)^{t S^{1}}$, respectively. We model $V(1)_{*} T F(\ell / p ; p)$ in degrees $>(2 p-2)$ by the map $\hat{\Gamma}$ to the $S^{1}$-Tate construction. Then, by diagram (6.1), $R_{*}$ is modeled in the same range of degrees by the chain of maps below:

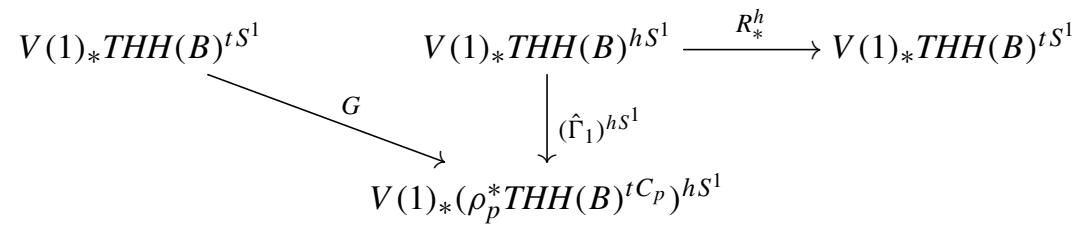

Here $R^{h}$ induces a map of spectral sequences

$$
E^{*}\left(R^{h}\right): E^{*}\left(S^{1}, B\right) \rightarrow \hat{E}^{*}\left(S^{1}, B\right)
$$

(abutting to $R_{*}^{h}$ ), which at the $E^{2}$-term equals the inclusion that algebraically inverts $t$. When $B=\ell / p$, the left hand map $G$ is an isomorphism by Lemma 6.8, and the middle (wrong-way) map is $(2 p-2)$-coconnected.

Proposition 7.1. In degrees $>2 p-2$, the homomorphism

$$
E^{\infty}\left(R^{h}\right): E^{\infty}\left(S^{1}, \ell / p\right) \rightarrow \hat{E}^{\infty}\left(S^{1}, \ell / p\right)
$$

maps

(a) $E\left(\bar{\epsilon}_{1}, \lambda_{2}\right) \otimes P\left(t \mu_{2}\right)$ identically to the same expression;

(b) $E\left(\lambda_{2}\right) \otimes \mathbb{F}_{p}\left\{\mu_{2}^{-j}\right\} \otimes P_{\rho(2 k-1)}\left(t \mu_{2}\right)$ surjectively onto $E\left(\lambda_{2}\right) \otimes \mathbb{F}_{p}\left\{t^{j}\right\} \otimes P_{\rho(2 k-3)}\left(t \mu_{2}\right)$ for each $k \geq 2, j=d p^{2 k-2}, 0<d<p^{2}-p$ and $p \nmid d$;

(c) $E\left(\bar{\epsilon}_{1}\right) \otimes \mathbb{F}_{p}\left\{\lambda_{2} \mu_{2}^{-j}\right\} \otimes P_{\rho(2 k)}\left(t \mu_{2}\right)$ surjectively onto $E\left(\bar{\epsilon}_{1}\right) \otimes \mathbb{F}_{p}\left\{t^{j} \lambda_{2}\right\} \otimes P_{\rho(2 k-2)}\left(t \mu_{2}\right)$ for each $k \geq 2, j=d p^{2 k-1}$ and $0<d<p$;

(d) the remaining terms to zero.

Notice that in statements (b) and (c) above, we abuse notation and identify the components of degree $>2 p-2$ of $E^{\infty}\left(S^{1}, \ell / p\right)$ and $\mu_{2}^{-1} E^{\infty}\left(S^{1}, \ell / p\right)$, using Theorem 6.9(b). 
Proof. Consider the summands of $E^{\infty}\left(S^{1}, \ell / p\right)$ and $\hat{E}^{\infty}\left(S^{1}, \ell / p\right)$ given in Theorem 6.9. Clearly, the first term $E\left(\lambda_{2}\right) \otimes \mathbb{F}_{p}\left\{\mu_{0}^{i} \mid 0<i<p\right\} \otimes P\left(\mu_{2}\right)$ goes to zero (these classes are hit by $d^{2}$-differentials), and the last term $E\left(\bar{\epsilon}_{1}, \lambda_{2}\right) \otimes P\left(t \mu_{2}\right)$ maps identically to the same term. This proves (a) and part of (d).

For each $k \geq 1$ and $j=d p^{2 k-2}$ with $p \nmid d$, the term $E\left(\lambda_{2}\right) \otimes \mathbb{F}_{p}\left\{\mu_{2}^{-j}\right\} \otimes P_{\rho(2 k-1)}\left(t \mu_{2}\right)$ maps to the term $E\left(\lambda_{2}\right) \otimes \mathbb{F}_{p}\left\{t^{j}\right\} \otimes P_{\rho(2 k-3)}\left(t \mu_{2}\right)$, except that the target is zero for $k=1$. In symbols, the element $\lambda_{2}^{\delta} \mu_{2}^{-j}\left(t \mu_{2}\right)^{i}$ maps to $\lambda_{2}^{\delta} t^{j}\left(t \mu_{2}\right)^{i-j}$. If $d<0$, then the $t$-exponent in the target is bounded above by $d p^{2 k-2}+\rho(2 k-3)<0$, so the target lives in the right half-plane and is essentially not hit by the source, which lives in the left half-plane. If $d>p^{2}-p$, then the total degree in the source is bounded above by $\left(2 p^{2}-1\right)-2 d p^{2 k}+\rho(2 k-1)\left(2 p^{2}-2\right)<2 p-2$, so the source lives in total degree $<2 p-2$ and will be disregarded. If $0<d<p^{2}-p$, then $\rho(2 k-1)-d p^{2 k-2}>\rho(2 k-3)$ and $-d p^{2 k-2}<0$, so the source surjects onto the target. This proves (b) and part of (d).

Lastly, for each $k \geq 1$ and $j=d p^{2 k-1}$ with $p \nmid d$, the term $E\left(\bar{\epsilon}_{1}\right) \otimes \mathbb{F}_{p}\left\{\lambda_{2} \mu_{2}^{-j}\right\} \otimes$ $P_{\rho(2 k)}\left(t \mu_{2}\right)$ maps to the term $E\left(\bar{\epsilon}_{1}\right) \otimes \mathbb{F}_{p}\left\{t^{j} \lambda_{2}\right\} \otimes P_{\rho(2 k-2)}\left(t \mu_{2}\right)$. The target is zero for $k=1$. If $d<0$, then $d p^{2 k-1}+\rho(2 k-2)<0$ so the target lives in the right half-plane. If $d>p$, then $(2 p-1)+\left(2 p^{2}-1\right)-2 d p^{2 k+1}+\rho(2 k)\left(2 p^{2}-2\right)<2 p-2$, so the source lives in total degree $<2 p-2$. If $0<d<p$, then $\rho(2 k)-d p^{2 k-1}>\rho(2 k-2)$ and $-d p^{2 k-1}<0$, so the source surjects onto the target. This proves (c) and the remaining part of (d).

Definition 7.2. Let

$$
\begin{aligned}
A & =E\left(\bar{\epsilon}_{1}, \lambda_{2}\right) \otimes P\left(t \mu_{2}\right), \\
B_{k} & =E\left(\lambda_{2}\right) \otimes \mathbb{F}_{p}\left\{t^{d p^{2 k-2}} \mid 0<d<p^{2}-p, p \nmid d\right\} \otimes P_{\rho(2 k-3)}\left(t \mu_{2}\right), \\
C_{k} & =E\left(\bar{\epsilon}_{1}\right) \otimes \mathbb{F}_{p}\left\{t^{d p^{2 k-1}} \lambda_{2} \mid 0<d<p\right\} \otimes P_{\rho(2 k-2)}\left(t \mu_{2}\right)
\end{aligned}
$$

for $k \geq 2$ and let $D$ be the span of the remaining monomials in $\hat{E}^{\infty}\left(S^{1}, \ell / p\right)$. Let $B=$ $\bigoplus_{k \geq 2} B_{k}$ and $C=\bigoplus_{k \geq 2} C_{k}$. Then $\hat{E}^{\infty}\left(S^{1}, \ell / p\right)=A \oplus B \oplus C \oplus D$.

Proposition 7.3. In degrees $>2 p-2$, there are closed subgroups $\widetilde{A}=E\left(\bar{\epsilon}_{1}, \lambda_{2}\right) \otimes$ $P\left(v_{2}\right), \widetilde{B}_{k}, \widetilde{C}_{k}$ and $\widetilde{D}$ in $V(1)_{*} T F(\ell / p ; p)$, represented by the subgroups $A, B_{k}, C_{k}$ and $D$ of $\hat{E}^{\infty}\left(S^{1}, \ell / p\right)$, respectively, such that the homomorphism $R_{*}=V(1)_{*} R$ induced by the restriction map $R$

(a) is the identity on $\widetilde{A}$;

(b) maps $\widetilde{B}_{k+1}$ surjectively onto $\widetilde{B}_{k}$ for all $k \geq 2$;

(c) maps $\widetilde{C}_{k+1}$ surjectively onto $\widetilde{C}_{k}$ for all $k \geq 2$;

(d) is zero on $\widetilde{B}_{2}, \widetilde{C}_{2}$ and $\widetilde{D}$.

In these degrees, $V(1)_{*} T F(\ell / p ; p) \cong \widetilde{A} \oplus \widetilde{B} \oplus \widetilde{C} \oplus \widetilde{D}$, where $\widetilde{B}=\prod_{k \geq 2} \widetilde{B}_{k}$ and $\widetilde{C}=\prod_{k \geq 2} \widetilde{C}_{k}$. 
Proof. The proof is the same as the proof of [AR02, Th. 7.7], except that in the present paper we work with the Tate model $T H H(\ell / p)^{t S^{1}}$ for $T F(\ell / p ; p)$, in place of the homotopy fixed point model $T H H(\ell / p)^{h S^{1}}$. The computations are made in $V(1)$-homotopy, and we disregard all classes in total degrees $\leq 2 p-2$. For example with this convention we write $\mu_{2}^{-1} E^{\infty}\left(S^{1}, \ell / p\right) \cong E^{\infty}\left(S^{1}, \ell / p\right)$, using the same abuse of notation as in Proposition 7.1.

In these terms, the restriction homomorphism $R_{*}$ is given at the level of $E^{\infty}$-terms as the composite of the isomorphism

$$
G_{*}: \hat{E}^{\infty}\left(S^{1}, \ell / p\right) \rightarrow \mu_{2}^{-1} E^{\infty}\left(S^{1}, \ell / p\right) \cong E^{\infty}\left(S^{1}, \ell / p\right)
$$

and the map

$$
E^{\infty}\left(R^{h}\right): E^{\infty}\left(S^{1}, \ell / p\right) \rightarrow \hat{E}^{\infty}\left(S^{1}, \ell / p\right) .
$$

As an endomorphism of $\hat{E}^{\infty}\left(S^{1}, \ell / p\right)$, this composite $E^{\infty}\left(R^{h}\right) G_{*}$ is the identity on $A$, maps $B_{k+1}$ onto $B_{k}$ and $C_{k+1}$ onto $C_{k}$ for all $k \geq 2$, and is zero on $B_{2}, C_{2}$ and $D$, by Theorem 6.9(c) and Proposition 7.1. The task is to find closed lifts of these groups to $V(1)_{*} T F(\ell / p ; p)$ such that $R_{*}$ has similar properties.

Let $\widetilde{A}=E\left(\bar{\epsilon}_{1}, \lambda_{2}\right) \otimes P\left(v_{2}\right) \subset V(1)_{*} T F(\ell / p ; p)$ be the (degreewise finite, hence closed) subalgebra generated by the images of the classes $\bar{\epsilon}_{1}^{K}, \lambda_{2}$ and $v_{2}$ in $V(1)_{*} K(\ell / p)$. Then $\widetilde{A}$ lifts $A$ and consists of classes in the image of the trace map from $V(1)_{*} K(\ell / p)$. Hence $R_{*}$ is the identity on $\widetilde{A}$.

We fix $k \geq 2$ and choose, for all $n \geq 0$, a subgroup $B_{k}^{n} \subset B_{k+n}$, as follows. We take

$$
\begin{aligned}
B_{k}^{0} & =B_{k} \cap \operatorname{ker}\left(E^{\infty}\left(R^{h}\right) G_{*}\right) \\
& = \begin{cases}B_{2} & \text { for } k=2, \\
E\left(\lambda_{2}\right) \otimes \underset{0<d<p^{2}-p, p \nmid d}{\bigoplus_{p}} \mathbb{F}_{p}\left\{t^{\left.d p^{2 k-2}\right\} \otimes P_{d p^{2 k-4}+\rho(2 k-5)}^{\rho(2 k-3)-1}\left(t \mu_{2}\right)}\right. & \text { for } k \geq 3,\end{cases}
\end{aligned}
$$

where $P_{a}^{b}\left(t \mu_{2}\right)=\mathbb{F}_{p}\left\{\left(t \mu_{2}\right)^{c} \mid a \leq c \leq b\right\}$. We proceed by induction on $n$ for $n \geq 1$, choosing a subgroup $B_{k}^{n}$ of $B_{k+n}$ mapping isomorphically onto $B_{k}^{n-1}$ under $E^{\infty}\left(R^{h}\right) G_{*}$ (such a group exists by Theorem 6.9(c) and Proposition 7.1(b)). We then have

$$
B_{k}=\bigoplus_{n=0}^{k-2} B_{k-n}^{n} .
$$

By the argument given on top of page 31 of [AR02], we can choose a lift $\widetilde{B}_{k}^{0}$ of $B_{k}^{0}$ with

$$
\widetilde{B}_{k}^{0} \subset \operatorname{im}\left(R_{*}\right) \cap \operatorname{ker}\left(R_{*}\right)
$$

in $V(1)_{*} T F(\ell / p ; p)$. By induction on $n \geq 1$, we choose a lift $\widetilde{B}_{k}^{n} \subset \operatorname{im}\left(R_{*}\right)$ of $B_{k}^{n}$ mapping isomorphically onto $\widetilde{B}_{k}^{n-1}$ under $R_{*}$. Such a choice is possible since the image 
of $R_{*}$ on $V(1)_{*} T F(\ell / p ; p)$ equals the image of its restriction to $\operatorname{im}\left(R_{*}\right)$ (see [AR02, p. 30]). Now

$$
\widetilde{B}_{k}=\bigoplus_{n=0}^{k-2} \widetilde{B}_{k-n}^{n}
$$

is a (degreewise finite, hence closed) lift of $B_{k}$ with $R_{*}\left(\widetilde{B}_{2}\right)=0$ and $R_{*}\left(\widetilde{B}_{k}\right)=\widetilde{B}_{k-1}$ for $k \geq 3$.

To construct $\widetilde{C}_{k}$ we proceed as for $\widetilde{B}_{k}$ above, starting with $C_{2}^{0}=C_{2}$ and

$$
\begin{aligned}
C_{k}^{0} & =C_{k} \cap \operatorname{ker}\left(E^{\infty}\left(R^{h}\right) G_{*}\right) \\
& =E\left(\bar{\epsilon}_{1}\right) \otimes \bigoplus_{0<d<p} \mathbb{F}_{p}\left\{t^{d p^{2 k-1}} \lambda_{2}\right\} \otimes P_{d p^{2 k-3}+\rho(2 k-4)}^{\rho(2 k-2)-1}\left(t \mu_{2}\right)
\end{aligned}
$$

for $k \geq 3$, and using Theorem 6.9(c) and Proposition 7.1(c) to choose $C_{k}^{n}$ for $n \geq 1$.

It remains to construct $\widetilde{D}$. By Proposition 7.1(d), the isomorphism $G_{*}$ maps $D$ into $\operatorname{ker}\left(E^{\infty}\left(R^{h}\right)\right)$. By [AR02, Lem. 7.3] the representatives in $E^{\infty}\left(S^{1}, \ell / p\right)$ of the kernel of $R_{*}^{h}$ equal the kernel of $E^{\infty}\left(R^{h}\right)$. It follows that the representatives in $\hat{E}^{\infty}\left(S^{1}, \ell / p\right)$ of the kernel of $R_{*}$ are mapped isomorphically by $G_{*}$ to $\operatorname{ker}\left(E^{\infty}\left(R^{h}\right)\right)$. Hence we can pick a vector space basis for $D$, choose a representative in $\operatorname{ker}\left(R_{*}\right) \subset V(1)_{*} T F(\ell / p ; p)$ of each basis element, and let $\widetilde{D} \subset V(1)_{*} T F(\ell / p ; p)$ be the closure of the vector space spanned by these chosen representatives. This closure is contained in $\operatorname{ker}\left(R_{*}\right)$ since $R_{*}$ is continuous. Hence $R_{*}$ is zero on $\widetilde{D}$.

Proposition 7.4. In degrees $>2 p-2$ there are isomorphisms

$$
\begin{aligned}
\operatorname{ker}\left(R_{*}-1\right) \cong & \widetilde{A} \oplus \lim _{k} \widetilde{B}_{k} \oplus \lim _{k} \widetilde{C}_{k} \\
\cong & E\left(\bar{\epsilon}_{1}, \lambda_{2}\right) \otimes P\left(v_{2}\right) \\
& \oplus E\left(\lambda_{2}\right) \otimes \mathbb{F}_{p}\left\{t^{d} \mid 0<d<p^{2}-p, p \nmid d\right\} \otimes P\left(v_{2}\right) \\
& \oplus E\left(\bar{\epsilon}_{1}\right) \otimes \mathbb{F}_{p}\left\{t^{d p} \lambda_{2} \mid 0<d<p\right\} \otimes P\left(v_{2}\right)
\end{aligned}
$$

and $\operatorname{cok}\left(R_{*}-1\right) \cong \widetilde{A}=E\left(\bar{\epsilon}_{1}, \lambda_{2}\right) \otimes P\left(v_{2}\right)$. Hence there is an isomorphism

$$
\begin{aligned}
V(1)_{*} T C(\ell / p ; p) \cong & E\left(\partial, \bar{\epsilon}_{1}, \lambda_{2}\right) \otimes P\left(v_{2}\right) \\
& \oplus E\left(\lambda_{2}\right) \otimes \mathbb{F}_{p}\left\{t^{d} \mid 0<d<p^{2}-p, p \nmid d\right\} \otimes P\left(v_{2}\right) \\
& \oplus E\left(\bar{\epsilon}_{1}\right) \otimes \mathbb{F}_{p}\left\{t^{d p} \lambda_{2} \mid 0<d<p\right\} \otimes P\left(v_{2}\right)
\end{aligned}
$$

in these degrees, where $\partial$ has degree -1 and represents the image of 1 under the connecting map д in (7.1).

Proof. By Proposition 7.3, the homomorphism $R_{*}-1$ is zero on $\widetilde{A}$ and an isomorphism on $\widetilde{D}$. Furthermore, there is an exact sequence

$$
0 \rightarrow \lim _{k} \widetilde{B}_{k} \rightarrow \prod_{k \geq 2} \widetilde{B}_{k} \stackrel{R_{*}-1}{\longrightarrow} \prod_{k \geq 2} \widetilde{B}_{k} \rightarrow \lim _{k}^{1} \widetilde{B}_{k} \rightarrow 0
$$

and similarly for the $C$ 's. The derived limit on the right vanishes since each $\widetilde{B}_{k+1}$ surjects onto $\widetilde{B}_{k}$. 
Multiplication by $t \mu_{2}$ in each $B_{k}$ is realized by multiplication by $v_{2}$ in $\widetilde{B}_{k}$. Each $\widetilde{B}_{k}$ is a sum of $2(p-1)^{2}$ cyclic $P\left(v_{2}\right)$-modules, and since $\rho(2 k-3)$ grows to infinity with $k$ their limit is a free $P\left(v_{2}\right)$-module of the same rank, with the indicated generators $t^{d}$ and $t^{d} \lambda_{2}$ for $0<d<p^{2}-p, p \nmid d$. The argument for the $C$ 's is practically the same.

The long exact sequence (7.1) yields the short exact sequence

$$
0 \rightarrow \Sigma^{-1} \operatorname{cok}\left(R_{*}-1\right) \stackrel{\partial}{\rightarrow} V(1)_{*} T C(\ell / p ; p) \stackrel{\pi}{\rightarrow} \operatorname{ker}\left(R_{*}-1\right) \rightarrow 0,
$$

from which the formula for the middle term follows.

Remark 7.5. A more obvious set of $E\left(\lambda_{2}\right) \otimes P\left(v_{2}\right)$-module generators for $\lim _{k} \widetilde{B}_{k}$ would be the classes $t^{d p^{2}}$ in $B_{2} \cong \widetilde{B}_{2}$, for $0<d<p^{2}-p, p \nmid d$. We have a commutative diagram

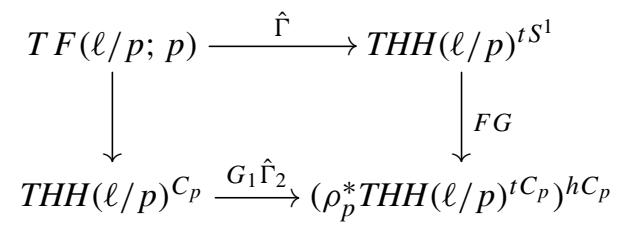

Under the left-hand canonical map $T F(\ell / p ; p) \rightarrow T H H(\ell / p)^{C_{p}}$, modelled here by

$$
F G: T H H(\ell / p)^{t S^{1}} \rightarrow\left(\rho_{p}^{*} T H H(\ell / p)^{t C_{p}}\right)^{h S^{1}} \rightarrow\left(\rho_{p}^{*} T H H(\ell / p)^{t C_{p}}\right)^{h C_{p}},
$$

the class $t^{d p^{2}}$ maps to $\mu_{2}^{-d}$. Since we are only concerned with degrees $>2 p-2$ we may equally well use its $v_{2}$-power multiple $\left(t \mu_{2}\right)^{d} \cdot \mu_{2}^{-d}=t^{d}$ as generator, with the advantage that it is in the image of the localization map

$$
T H H(\ell / p)^{h C_{p}} \rightarrow\left(\rho_{p}^{*} T H H(\ell / p)^{t C_{p}}\right)^{h C_{p}} .
$$

Hence the class denoted $t^{d}$ in $\lim _{k} \widetilde{B}_{k}$ is chosen so as to map under $T F(\ell / p ; p) \rightarrow$ $T H H(\ell / p)^{h C_{p}}$ to $t^{d}$ in $E^{\infty}\left(C_{p}, \ell / p\right)$. Similarly, the class denoted $t^{d p} \lambda_{2}$ in $\lim _{k} \widetilde{C}_{k}$ is chosen so as to map to $t^{d p} \lambda_{2}$ in $E^{\infty}\left(C_{p}, \ell / p\right)$.

The map $\pi: \ell / p \rightarrow \mathbb{Z} / p$ is $(2 p-2)$-connected, hence induces $(2 p-1)$-connected maps $\pi_{*}: K(\ell / p) \rightarrow K(\mathbb{Z} / p)$ and $\pi_{*}: V(1)_{*} T C(\ell / p ; p) \rightarrow V(1)_{*} T C(\mathbb{Z} / p ; p)$, by [BM94, Prop. 10.9] and [Dun97, p. 224]. Here $T C(\mathbb{Z} / p ; p) \simeq H \mathbb{Z}_{p} \vee \Sigma^{-1} H \mathbb{Z}_{p}$ and we have an isomorphism $V(1)_{*} T C(\mathbb{Z} / p ; p) \cong E\left(\partial, \bar{\epsilon}_{1}\right)$, so we can recover $V(1)_{*} T C(\ell / p ; p)$ in degrees $\leq 2 p-2$ from this map.

Theorem 7.6. There is an isomorphism of $P\left(v_{2}\right)$-modules

$$
\begin{aligned}
V(1)_{*} T C(\ell / p ; p) \cong & P\left(v_{2}\right) \otimes E\left(\partial, \bar{\epsilon}_{1}, \lambda_{2}\right) \\
& \oplus P\left(v_{2}\right) \otimes E\left(\operatorname{dlog} v_{1}\right) \otimes \mathbb{F}_{p}\left\{t^{d} v_{2} \mid 0<d<p^{2}-p, p \nmid d\right\} \\
& \oplus P\left(v_{2}\right) \otimes E\left(\bar{\epsilon}_{1}\right) \otimes \mathbb{F}_{p}\left\{t^{d p} \lambda_{2} \mid 0<d<p\right\}
\end{aligned}
$$

where $\operatorname{dog} v_{1} \cdot t^{d} v_{2}=t^{d} \lambda_{2}$. The degrees are $|\partial|=-1,\left|\bar{\epsilon}_{1}\right|=\left|\lambda_{1}\right|=2 p-1,\left|\lambda_{2}\right|=$ $2 p^{2}-1,\left|v_{2}\right|=2 p^{2}-2,|t|=-2$ and $\left|\operatorname{dlog} v_{1}\right|=1$. 
The notation d $\log v_{1}$ for the multiplier $v_{2}^{-1} \lambda_{2}$ is suggested by the relation $v_{1} \cdot \operatorname{dlog} p=\lambda_{1}$ in $V(0)_{*} T C\left(\mathbb{Z}_{(p)} \mid \mathbb{Q} ; p\right)$.

Proof. Only the additive generators $t^{d}$ for $0<d<p^{2}-p, p \nmid d$ from Proposition 7.4 do not appear in $V(1)_{*} T C(\ell / p ; p)$, but their multiples by $\lambda_{2}$ and positive powers of $v_{2}$ do. This leads to the given formula, where $\operatorname{dlog} v_{1} \cdot t^{d} v_{2}$ must be read as $t^{d} \lambda_{2}$.

By [HM97, Th. C] the cyclotomic trace map of [BHM93] induces cofibre sequences

$$
K\left(B_{p}\right)_{p} \stackrel{\operatorname{trc}}{\rightarrow} T C(B ; p)_{p} \stackrel{g}{\rightarrow} \Sigma^{-1} H \mathbb{Z}_{p} \rightarrow \Sigma K\left(B_{p}\right)_{p}
$$

for each connective $S$-algebra $B$ with $\pi_{0}\left(B_{p}\right)=\mathbb{Z}_{p}$ or $\mathbb{Z} / p$, and thus long exact sequences

$$
\cdots \rightarrow V(1)_{*} K\left(B_{p}\right) \stackrel{\operatorname{trc}}{\rightarrow} V(1)_{*} T C(B ; p) \stackrel{g}{\rightarrow} \Sigma^{-1} E\left(\bar{\epsilon}_{1}\right) \rightarrow \cdots .
$$

This uses the identifications $W\left(\mathbb{Z}_{p}\right)_{F} \cong W(\mathbb{Z} / p)_{F} \cong \mathbb{Z}_{p}$ of Frobenius coinvariants of rings of Witt vectors, and applies in particular for $B=H \mathbb{Z}_{(p)}, H \mathbb{Z} / p, \ell$ and $\ell / p$.

Theorem 7.7. There is an isomorphism of $P\left(v_{2}\right)$-modules

$$
\begin{aligned}
V(1)_{*} K(\ell / p) \cong & P\left(v_{2}\right) \otimes E\left(\bar{\epsilon}_{1}\right) \otimes \mathbb{F}_{p}\left\{1, \partial \lambda_{2}, \lambda_{2}, \partial v_{2}\right\} \\
& \oplus P\left(v_{2}\right) \otimes E\left(\operatorname{dlog} v_{1}\right) \otimes \mathbb{F}_{p}\left\{t^{d} v_{2} \mid 0<d<p^{2}-p, p \nmid d\right\} \\
& \oplus P\left(v_{2}\right) \otimes E\left(\bar{\epsilon}_{1}\right) \otimes \mathbb{F}_{p}\left\{t^{d p} \lambda_{2} \mid 0<d<p\right\} .
\end{aligned}
$$

This is a free $P\left(v_{2}\right)$-module of rank $2 p^{2}-2 p+8$ and of zero Euler characteristic.

Proof. In the case $B=\mathbb{Z} / p, K(\mathbb{Z} / p)_{p} \simeq H \mathbb{Z}_{p}$ and the map $g$ is split surjective up to homotopy. So the induced homomorphism to $V(1)_{*} \Sigma^{-1} H \mathbb{Z}_{p}=\Sigma^{-1} E\left(\bar{\epsilon}_{1}\right)$ is surjective. Since $\pi: \ell / p \rightarrow \mathbb{Z} / p$ induces a (2p-1)-connected map in topological cyclic homology, and $\Sigma^{-1} E\left(\bar{\epsilon}_{1}\right)$ is concentrated in degrees $\leq 2 p-2$, it follows by naturality that also in the case $B=\ell / p$ the map $g$ induces a surjection in $V(1)$-homotopy. The kernel of the surjection $P\left(v_{2}\right) \otimes E\left(\partial, \bar{\epsilon}_{1}, \lambda_{2}\right) \rightarrow \Sigma^{-1} E\left(\bar{\epsilon}_{1}\right)$ gives the first row in the asserted formula.

Acknowledgments. We would like to thank the referee for his many useful suggestions. Both authors were partially supported by the Max Planck Institute for Mathematics, Bonn. The first author was partially supported by the DFG Leibniz Award of Prof. W. Lück.

\section{References}

[Ang08] Angeltveit, V.: Topological Hochschild homology and cohomology of $A_{\infty}$ ring spectra. Geom. Topol. 12, 987-1032 (2008) Zbl 1149.55006 MR 2403804

[Ang11] Angeltveit, V.: Uniqueness of Morava $K$-theory. Compos. Math. 147, 633-648 (2011) Zbl 1220.55004 MR 2776615

[AR05] Angeltveit, V., Rognes, J.: Hopf algebra structure on topological Hochschild homology. Algebr. Geom. Topol. 5, 1223-1290 (2005) Zbl 1087.55009 MR 2171809 
[Aus05] Ausoni, Ch.: Topological Hochschild homology of connective complex $K$-theory. Amer. J. Math. 127, 1261-1313 (2005) Zbl 1107.55006 MR 2183525

[Aus10] Ausoni, Ch.: On the algebraic $K$-theory of the connective complex $K$-theory spectrum. Invent. Math. 180, 611-668 (2010) Zbl 1204.19002 MR 2609252

[AR02] Ausoni, Ch., Rognes, J.: Algebraic $K$-theory of topological $K$-theory. Acta Math. 188, 1-39 (2002) Zbl 1019.18008 MR 1947457

[AR] Ausoni, Ch., Rognes, J.: Algebraic $K$-theory of the fraction field of topological $K$ theory. arXiv:0911.4781.

[BJ02] Baker, A., Jeanneret, A.: Brave new Hopf algebroids and extensions of $M U$-algebras. Homology Homotopy Appl. 4, 163-173 (2002) MR 1937961

[BR05] Baker, A., Richter, B.: On the $\Gamma$-cohomology of rings of numerical polynomials and $E_{\infty}$ structures on $K$-theory. Comment. Math. Helv. 80, 691-723 (2005) Zbl 1094.55010 MR 2182697

[Ben98] Benson, D. J.: Representations and Cohomology. I. 2nd ed., Cambridge Univ. Press, Cambridge (1998) Zbl 0908.20001 MR 1644252

[BM08] Blumberg, A. J., Mandell, M. A.: The localization sequence for the algebraic $K$ theory of topological $K$-theory. Acta Math. 200, 155-179 (2008) Zbl 1149.18008 MR 2413133

[Bök] Bökstedt, M.: Topological Hochschild homology of $\mathbb{Z}$ and $\mathbb{Z} / p$. Unpublished preprint.

[BBLNR] Bökstedt, M., Bruner, B., Lunøe-Nielsen, S., Rognes, J.: On cyclic fixed points of spectra. arXiv:0712.3476.

[BHM93] Bökstedt, M., Hsiang, W. C., Madsen, I.: The cyclotomic trace and algebraic $K$-theory of spaces. Invent. Math. 111, 465-539 (1993) Zbl 0804.55004 MR 1202133

[BM94] Bökstedt, M., Madsen, I.: Topological cyclic homology of the integers. In: $K$-theory (Strasbourg, 1992), Astérisque 226, 57-143 (1994) Zbl 0816.19001 MR 1317117

[BM95] Bökstedt, M., Madsen, I.: Algebraic $K$-theory of local number fields: the unramified case. In: Prospects in Topology (Princeton, NJ, 1994), Ann. of Math. Stud. 138, Princeton Univ. Press, Princeton, NJ, 28-57 (1995) Zbl 0924.19001 MR 1368652

[BMMS86] Bruner, R. R., May, J. P., McClure, J. E., Steinberger, M.: $H_{\infty}$ Ring Spectra and Their Applications. Lecture Notes in Math. 1176, Springer, Berlin (1986) Zbl 0585.55016 MR 0836132

[Dun97] Dundas, B. I.: Relative $K$-theory and topological cyclic homology. Acta Math. 179, 223-242 (1997) Zbl 0913.19002 MR 1607556

[Dun04] Dundas, B. I.: The cyclotomic trace for $S$-algebras. J. London Math. Soc. (2) 70, 659677 (2004) Zbl 1069.19005 MR 2096869

[EKMM97] Elmendorf, A. D., Kriz, I., Mandell, M. A., May, J. P.: Rings, Modules, and Algebras in Stable Homotopy Theory, with an appendix by M. Cole. Math. Surveys Monogr. 47, Amer. Math. Soc., Providence, RI (1997) Zbl 0894.55001 MR 1417719

[GM95] Greenlees, J. P. C., May, J. P.: Generalized Tate cohomology. Mem. Amer. Math. Soc. 113, no. 543 (1995) Zbl 0876.55003 MR 1230773

[HM97] Hesselholt, L., Madsen, I.: On the $K$-theory of finite algebras over Witt vectors of perfect fields. Topology 36, 29-101 (1997) Zbl 0866.5500 MR 1410465

[HM03] Hesselholt, L., Madsen, I.: On the $K$-theory of local fields. Ann. of Math. (2) 158, 1-113 (2003) Zbl 1033.19002 MR 1998478

[Hun96] Hunter, T. J.: On the homology spectral sequence for topological Hochschild homology. Trans. Amer. Math. Soc. 348, 3941-3953 (1996) Zbl 0869.55017 MR 1376548

[Laz01] Lazarev, A.: Homotopy theory of $A_{\infty}$ ring spectra and applications to $M U$-modules. $K$-Theory 24, 243-281 (2001) Zbl 1008.55007 MR 1876800 
[Lod98] Loday, J.-L.: Cyclic Homology. Springer, Berlin (1998) Zbl 0885.18007 MR 1600246

[MS93] McClure, J. E., Staffeldt, R. E.: On the topological Hochschild homology of $b u$, I. Amer. J. Math. 115, 1-45 (1993) Zbl 0770.55010 MR 1209233

[Mil58] Milnor, J.: The Steenrod algebra and its dual. Ann. of Math. (2) 67, 150-171 (1958) Zbl 0080.38003 MR 0099653

[MM65] Milnor, J. W., Moore, J. C.: On the structure of Hopf algebras. Ann. of Math. (2) 81, 211-264 (1965) Zbl 0163.28202 MR 0174052

[Oka84] Oka, S.: Multiplicative structure of finite ring spectra and stable homotopy of spheres. In: Algebraic Topology (Aarhus, 1982), Lecture Notes in Math. 1051, Springer, Berlin, 418-441 (1984) Zbl 0545.55013 MR 0764594

[Qui72] Quillen, D.: On the cohomology and $K$-theory of the general linear groups over a finite field. Ann. of Math. (2) 96, 552-586 (1972) Zbl 0249.18022 MR 0315016

[Rog98] Rognes, J.: Trace maps from the algebraic $K$-theory of the integers (after Marcel Bökstedt). J. Pure Appl. Algebra 125, 277-286 (1998) Zbl 0893.19001 MR 1600028

[Rog08] Rognes, J.: Galois extensions of structured ring spectra. Mem. Amer. Math. Soc. 192, no. 898, 97 pp. (2008) Zbl 1166.55001 MR 2387923

[Smi70] Smith, L.: On realizing complex bordism modules. Applications to the stable homotopy of spheres. Amer. J. Math. 92, 793-856 (1970) Zbl 0218.55023 MR 0275429

[Tod71] Toda, H.: On spectra realizing exterior parts of the Steenrod algebra. Topology 10, 53-65 (1971) Zbl 0223.55029 MR 0271933

[Tsa98] Tsalidis, S.: Topological Hochschild homology and the homotopy descent problem. Topology 37, 913-934 (1998) Zbl 0922.19001 MR 1607764

[Whi62] Whitehead, G. W.: Generalized homology theories. Trans. Amer. Math. Soc. 102, 227-283 (1962) Zbl 0124.38302 MR 0137117 